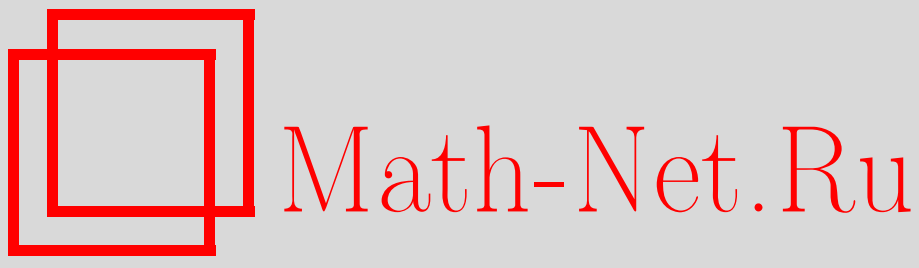

С. В. Востоков, Ф. Лоренц, Явная формула символа Гильберта для групп Хонды в многомерном локальном поле, Матем. сб., 2003, том 194, номер 2, 3-36

DOI: https://doi.org/10.4213/sm711

Использование Общероссийского математического портала Math-Net.Ru подразумевает, что вы прочитали и согласны с пользовательским соглашением http://www . mathnet.ru/rus/agreement

Параметры загрузки:

IP : 54.197 .217 .227

26 апреля 2023 г., 15:50:27 
УДК 512.741

\author{
С.В. Востоков, $\Phi$. Лоренц
}

\title{
Явная формула символа Гильберта для групп Хонды в многомерном локальном поле
}

\begin{abstract}
На основе построенного в явном виде в предыдущей статье авторов спаривания на кривых Картье конструируется явная формула для символа Гильберта в многомерном локальном поле нулевой характеристики с полем вычетов положительной характеристики на формальном модуле одномерной формальной группы Хонды. При доказательстве строится базис Шафаревича на формальном модуле и изучаются так называемые целые $\mu$-модули в специального вида двумерных локальных кольцах ( $\mu$-кольцах).

Библиографоия: 15 названий.
\end{abstract}

\section{Введение}

В статье [1] было построено в явном виде спаривание на модуле кривых Картье формальной группы Хонды в многомерном локальном поле. В настоящей работе на основе этого спаривания мы строим спаривание $\langle\cdot, \cdot\rangle_{F}$ на формальном модуле $\mathscr{H}_{F}$, проверяем его основные свойства и доказываем совпадение этого спаривания со спариванием Гильберта, что дает для последнего явную формулу.

Такие явные формулы для многомерных групп Хонды над кольцом векторов Витта были получены В. Абрашкиным в [2] при некоторых ограничениях, а для одномерных групп Хонды в произвольном неразветвленном расширении одномерного локального поля - в работе [3]. Подробную библиографию по явным формулам для символа Гильберта можно найти в [4] или [5].

Первый параграф̆ работы носит вспомогательный характер - в нем вводятся основные обозначения и определения.

Во втором параграфе строится спаривание $\langle\cdot, \cdot\rangle_{F}$ и формулируются основные результаты работы (теоремы 1 и 2 ).

В третьем параграфе мы проверяем корректность построенного спаривания $\langle\cdot, \cdot\rangle_{F}$ (см. п. 3.1); далее проверяем независимость спаривания $\langle\cdot, \cdot\rangle_{F}$ по второму аргументу от разложения элементов спаривания в ряды по локальным униформизуюшим (см. п. 3.2 ); в п. 3.3 вычисляем значения спаривания $\langle\cdot, \cdot\rangle_{F}$ на базисе Шафаревича; в следуюшем п. 3.4 доказываем инвариантность спаривания $\langle\cdot, \cdot\rangle_{F}$ от выбора системы локальных униформизуюших; и наконец, в последнем п. 3.5 третьего параграфа проверяем независимость нашего спаривания по первому аргументу.

Работа первого автора выполнена при поддержке Российского фонда фундаменталњных исследований (гранты № № 00-01-00140, 01-01-00997) и Министерства образования Р $\Phi$ (грант № Р Д02-1.1-37).

(C) С. В. Востоков, Ф. ЛОРенц 2003 
Основные теоремы 1 и 2 доказываются в $\S 4$.

Авторы благодарны Sonderforschungsbereich Geometrische Strukturen $\mathrm{У}_{\text {нивер- }}$ ситета г. Мюнстер за поддержку.

\section{§1. Обозначения и вспомогательные утверждения}

1.1. Пусть $k-n$-мерное локальное поле, т.е. конечная последовательность полей $k^{(0)}, k^{(1)}, \ldots, k^{(n)}=k$, где $k^{(0)}=\mathbb{F}_{q}, q=p^{f}$, и $k^{(i)}$ для $1 \leqslant i \leqslant n$ - полное дискретно нормированное поле, для которого $k^{(i-1)}$ является полем вычетов.

Интенсивное изучение таких полей началось в семидесятых годах в работах А. Паршина и К. Като. В настоящее время эти поля уже достаточно исследованы (см., например, [6]).

Для $n$-мерного локального поля $k$ имеют место следующие структуры:

1) дискретное нормирование $v: k^{*} \rightarrow \mathbb{Z}$ ранга 1 ;

2 ) дискретное нормирование $\bar{v}=\left(v_{1}, \ldots, v_{n-1}, v_{n}=v\right): k^{*} \rightarrow \mathbb{Z}^{n}$ ранга $n$.

Все $n$-мерные локальные поля, которые рассматриваются в статье, предполагаются нулевой характеристики, а простое число $p \neq 2$.

Пусть $\mathfrak{o}=\mathfrak{o}_{k}-$ кольцо целых относительно нормирования $\bar{v}$, а $t_{1}, \ldots, t_{n-1}$, $t_{n}=\pi$-система локальных параметров для $\bar{v}$. Через $\mathfrak{R}$ обозначаем систему представителей Тейхмюллера поля $\mathbb{F}_{q}$ в o. Произвольньй элемент $\alpha \in k$ имеет единственное разложение

$$
\alpha=\sum_{\bar{r} \in I} \theta_{\bar{r}} t_{1}^{r_{1}} \cdots t_{n-1}^{r_{n-1}} \pi^{r_{n}}, \quad \theta_{\bar{r}} \in \mathfrak{R}
$$

где $\bar{r}=\left(r_{1}, \ldots, r_{n}\right)$ пробегает некоторый допустимый набор индексов $I=I(\alpha)$ в $\mathbb{Z}^{n}$. При этом $I \subset \mathbb{Z}^{n}$ называем допустимылм, если для заданных $i_{l+1}, \ldots, i_{n}$ в $\mathbb{Z}, l \leqslant n$, сушествует $i \in \mathbb{Z}$, для которого выполнено следуюшее свойство: если $\bar{r}=\left(r_{1}, \ldots, r_{l}, i_{l+1}, \ldots, i_{n}\right) \in I$, то $r_{l} \geqslant i$ (см. [6]). Мы рассматриваем также разложения вида

$$
\alpha=\sum_{\bar{r} \in I} a_{\bar{r}} t_{1}^{r_{1}} \cdots t_{n-1}^{r_{n-1}} \pi^{r_{n}}
$$

с коэффициентами $a_{\bar{r}}$ в $\mathfrak{o}_{0}:=\mathrm{W}\left(\mathbb{F}_{q}\right)$, где $\mathrm{W}\left(\mathbb{F}_{q}\right)$ - кольцо векторов Витта над $\mathbb{F}_{q}$; это разложение уже не будет единственньп для $\alpha$.

1.2. Пусть $k^{\prime} / k$ - конечное неразветвленное расширение $n$-мерного локального поля $k$, и пусть $\Delta$ обозначает автоморфизм Фробениуса в $k^{\prime} / k$. Определим оператор Фробениуса $\boldsymbol{\Delta}$ на кольце $k^{\prime}((x))$ следующим образом:

$$
\mathbf{\Delta}: f(x)=\sum c_{i} x^{i} \mapsto \mathbf{\Delta} f=\sum c_{i}^{\Delta} x^{q i}
$$

Пусть $\mathfrak{o}^{\prime}$ - кольцо целых поля $k^{\prime}$ относительно $n$-мерного нормирования $\bar{v}^{\prime}$.

Обозначим через $\mathfrak{o}^{\prime}[[\mathbf{\Delta}]]$ некоммутативное кольцо степенных рядов $\sum a_{i} \boldsymbol{\Delta}^{i}$, $a_{i} \in \mathfrak{o}^{\prime}$, с правилом

$$
\mathbf{\Delta} a=a^{\Delta} \mathbf{\Delta}, \quad a \in \mathfrak{o}^{\prime}
$$


1.3. Пусть $k^{\prime} / k$ - неразветвленное расширение одномерного локального поля $k$ (конечное расширение $\mathbb{Q}_{p}$ ), и пусть $\mathfrak{m}^{\prime}$ - максимальный идеал кольца целых $\mathfrak{o}^{\prime}$ поля $k^{\prime}$. Одномерную формальную группу $F$ над $\mathfrak{o}^{\prime}$ называем группой Хонды, если существует специальный элемент

$$
u=\pi+a_{1} \mathbf{\Lambda}+a_{2} \mathbf{\Lambda}^{2}+\cdots \in \mathfrak{o}^{\prime}[[\mathbf{\Lambda}]]
$$

которьй для логарифма $\lambda:=\lambda(x)$ группы $F$ удовлетворяет условию

$$
(u \circ \lambda)(x) \equiv 0 \quad \bmod \pi
$$

(см. [7]). Если $u$ имеет это свойство, то будем говорить, что группа Хонды $F$ (и соответствуюший ей логарифм $\lambda$ ) имеет тuп $u$.

Ряд $u$ можно заменить на любой элемент $\widetilde{u}=\varepsilon u$, где $\varepsilon=1+b_{1} \mathbf{\Lambda}+\cdots \in \mathfrak{o}^{\prime}[[\mathbf{\Lambda}]]$. Поэтому по подготовительной лемме Вейерштрасса для группы $F$ можно получить следующий канонический тип:

$$
u_{0}= \begin{cases}\pi, & \text { если ht } F=\infty, \\ \pi-a_{1} \mathbf{\Delta}-\cdots-a_{h} \mathbf{\Delta}^{h}, & \text { если ht } F<\infty\end{cases}
$$

где $a_{1}, \ldots, a_{h-1} \in \mathfrak{m}^{\prime}, a_{h} \in \mathfrak{o}^{\prime \times}$ и ht $F$ - высота группы $F$. При этом ht $F$ будет равна $h$.

Этим каноническим типом группа $F$ однозначно определена (с точностью до изоморфизма). Кроме того, согласно [7; теорема 3$]$

$$
\operatorname{End}_{\mathfrak{o}^{\prime}}(F) \approx \mathfrak{o}
$$

Если $k=\mathbb{Q}_{p}$, то любая формальная группа над o' является группой Хонды.

Для групा Хонды над $\mathfrak{o}^{\prime}$ имеют место классификационные теоремы Демченко (см. [8]):

ПРЕДЛОЖЕНИЕ 1. Пусть $F$ - группа Хонды над $\mathfrak{o}^{\prime}$ с логарифмом $\lambda$ и каноническим типом $u_{0}($ см. (2)). Тогда $F$ имеет также тип

$$
\widetilde{u}:=C^{-1} u_{0}=\pi-a_{h} \mathbf{\Delta}^{h}-a_{h+1} \mathbf{\Delta}^{h+1}-\cdots,
$$

əде $C:=\pi^{-1}\left(u_{0}+a_{h} \mathbf{\Lambda}^{h}\right)$.

Запишем $\widetilde{u}=\pi-a_{h} B_{1} \mathbf{\Delta}^{h}$. Тогда

1) $\lambda_{1}=B_{1} \circ \lambda^{\Delta^{h}}-$ логарифм группы Хонды $F_{1}$ над $\mathfrak{o}^{\prime}$ mипа $\widetilde{u}_{1}=a_{h}^{-1} \widetilde{u} a_{h} u$ канонического типа $u_{1}:=a_{h}^{-1} u_{0} a_{h}$,

2) $f:=\left[\pi / a_{h}\right]_{F, F_{1}} \in \operatorname{Hom}_{\mathfrak{o}^{\prime}}\left(F, F_{1}\right)$, u $f(x) \equiv x^{q^{h}} \bmod \pi$. 
ПрЕДЛОЖЕНИЕ 2 (обратное к предложению 1). Пусть $f(x)-$ произвольныи ряд из $\mathfrak{o}^{\prime}[[\mathbf{\Delta}]]$, удовлетворяющий условиям

$$
f(x) \equiv x^{q^{h}} \quad \bmod \pi, \quad f(x) \equiv a_{h}^{-1} \pi \quad \bmod x^{2}
$$

с фиксированной единицей $a_{h}$ из $\mathfrak{o}^{\prime}$. Пусть, далее, задан $u_{0}=\pi-a_{1} \mathbf{\Delta}-\cdots-$ $a_{h} \mathbf{\Delta}^{h}, a_{1}, \ldots, a_{h-1} \in \mathfrak{m}^{\prime}$, u nycms $\widetilde{u}:=C^{-1} u_{0}$, где $C:=\pi^{-1}\left(u_{0}+a_{h} \mathbf{\Delta}^{h}\right)$.

Тогда существует единственная (с точностью до изоморфизма) группа Хонды $F$ типа $\widetilde{u}$ и канонического типа $u_{0}$ такая, что $f=\left[\pi / a_{h}\right]_{F, F_{1}}$ изогения $F$ в формальную группу Хондь $F_{1}$, которая определяется однозначно по $F$ в соответствии с предложсением 1.

Для группы Хонды над $\mathfrak{o}^{\prime}$ получаем отображение

$$
\mathscr{A}: F \mapsto F_{1}
$$

и последовательность гомоморфизмов $f_{m}$ групп Хонды $F_{m}:=\mathscr{A}^{m} F$ :

$$
F \longrightarrow F_{1} \longrightarrow f_{1} \longrightarrow F_{N-1} \underset{f_{N-1}}{\longrightarrow} F_{N}
$$

Обозначим через $\lambda_{m}$ логарифм $F_{m}$ и через $B_{m}$ - оператор из предложения 1 , который соответствует $\lambda_{m}$. Канонический тип $F_{m}$ обозначаем через

$$
u_{m}=\pi-a_{1}^{(m)} \mathbf{\Delta}-\ldots-a_{h}^{(m)} \mathbf{\Delta}^{h} .
$$

Кроме того, положим

$$
\begin{gathered}
\pi_{1}:=\frac{\pi}{a_{h}}, \quad \pi_{m}:=\pi_{1}^{\Delta^{h(m-1)}}=\frac{\pi}{a_{h}^{\Delta^{h(m-1)}}}, \\
\pi_{1}^{(m)}=\pi_{1} \pi_{2} \cdots \pi_{m}=\frac{\pi^{m}}{a_{h}^{1+\Delta^{h}+\cdots+\Delta^{h(m-1)}}}, \\
f^{(m)}=f_{m-1} \circ f_{m-2} \circ \cdots \circ f_{1} \circ f .
\end{gathered}
$$

1.4. Многомерный символ Гильберта для групп Хонды. Пусть $k_{c}-$ одномерное локальное поле, и предположим, что $k-n$-мерное локальное поле типа $k=k_{c}\left\{\left\{t_{1}\right\}\right\} \ldots\left\{\left\{t_{n-1}\right\}\right\}$. Пусть $k_{c}^{\prime}$ и $k^{\prime}$ - неразветвленные расшпрения полей $k_{c}$ и $k$ соответственно; пусть $\mathfrak{o}$ и $\mathfrak{m}-$ кольцо целых и максимальный идеал в $k_{c}$. Фиксируем группу Хонды $F$ над кольцом целых $\mathfrak{o}^{\prime}$ поля $k_{c}^{\prime}$.

Положим

$$
W_{F}^{N}:=\left\{\alpha \in F(\widehat{\mathfrak{M}}) ;\left[\pi^{N}\right]_{F}(\alpha)=0\right\}=\operatorname{Ker}\left[\pi^{N}\right]_{F},
$$

где $F(\widehat{\mathfrak{M}})$ - формальный о-модуль группы $F$ на максимальном идеале $\widehat{\mathfrak{M}}$ кольца целых пополнения $\widehat{\Omega}$ алгебраического замыкания поля $k$.

Обозначим далее:

$K$ - конечное расширение поля $k^{\prime}$, содержащее $W_{F}^{N}$;

$\bar{v}=\left(v_{1}, \ldots, v_{n}\right)-n$-мерное нормирование на $K$;

$\mathfrak{M}$ - максимальньй идеал относительно нормирования $\bar{v}$; 
$\Pi$ - простой элемент в $K$ относительно $v_{n}$;

$e$ - индекс ветвления $K / k$ относительно $v_{n}$;

$\bar{e}=\left(e_{1}, \ldots, e_{n}\right)-$ индекс ветвления $K / k$ относительно $\bar{v}$;

$\bar{e}_{i}=\bar{e} /\left(\left(q^{h}-1\right) q^{h(i-1)}\right)$ для $1 \leqslant i \leqslant N$;

$\bar{e}_{*}=\left(e_{1}^{*}, \ldots, e_{n}^{*}\right)=q^{h} \bar{e} /\left(q^{h}-1\right)$;

$T=k_{c}^{n r} \cap K$

$\mathfrak{o}_{T}-$ кольцо целых поля $T$;

$\operatorname{Tr}$ - оператор следа в $T / k_{c}$;

$T_{0}=\operatorname{Quot}\left(\mathrm{W}\left(K^{(0)}\right)\right)$ - поле отношений кольца векторов Витта, $\Delta_{0}, \Delta$ - автоморфизмы Фробениуса в $T_{0} / \mathbb{Q}_{p}, k^{\prime} / k$ соответственно; при этом $\left.\Delta\right|_{T_{0}}=\Delta_{0}^{f}$, где $p^{f}=q$;

$\Psi_{K}: K_{n}^{\mathrm{top}}(K) \rightarrow \operatorname{Gal}\left(K^{\mathrm{ab}} / K\right)$ - отображение взаимности à la Паршин-Като из топологической группы Милнора в группу Галуа максимального абелева расширения поля $K$ (см. [9]-[11]).

Модуль $W_{F}^{N}$ изоморфен $\left(\mathfrak{o} / \mathfrak{m}^{N}\right)^{h}$ и имеет, таким образом, $h$ образующих (см. [12; $\S 1]$ и [13; теорема 1]). Фиксируем систему образующих $z_{1}, \ldots, z_{h}$ o-модуля $W_{F}^{N}$. Для каждого $z=z_{i}$ имеем

$$
\bar{v}(z)=\frac{\bar{e}}{\left(q^{h}-1\right) q^{(N-1) h}}=\bar{e}_{N}
$$

(см. $[1 ; 1.5$, лемма 3$])$.

Пусть $\mathscr{H}_{F}-$ модуль кривых Картье для группы $F($ см. $[1 ; 1.8])$ и

$$
\begin{gathered}
\eta_{F}: \mathscr{H}_{F} \rightarrow F(\mathfrak{M}), \\
\alpha(X) \mapsto \alpha\left(t_{1}, \ldots, t_{n-1}, \Pi\right),
\end{gathered}
$$

- гомоморфизм о-модулей. Для каждого $\alpha \in F(\mathfrak{M})$ выбираем прообраз $\underline{\alpha} \in \mathscr{H}_{F}$ такой, что $\underline{\alpha}\left(t_{1}, \ldots, t_{n-1}, \Pi\right)=\alpha$.

Рассмотрим следующие элементы из $\mathfrak{o}_{T}[[X]]$ :

$$
\begin{gathered}
s_{m}:=f^{(m)} \circ \underline{z}, \quad 1 \leqslant m \leqslant N ; \\
\operatorname{Eis}_{F}(X):=s_{N} / s_{N-1}=V \circ \underline{z}, \quad \text { где } \quad V=f^{(N)} / f^{(N-1)} ; \\
W^{(m)}:=\pi_{1}^{(m)} \lambda \circ \underline{z}, \quad W:=W^{(N)}, \quad W_{i}:=\pi_{1}^{(N)} \lambda \circ \underline{z}_{i} .
\end{gathered}
$$

Определим многомерный символ Гильберта для группы Хонды $F$ следующим образом:

$$
\begin{aligned}
(\cdot, \cdot)= & (\cdot, \cdot)_{F, K}^{N}: K_{n}^{\mathrm{top}}(K) \times F(\mathfrak{M}) \rightarrow W_{F}^{N}, \\
& (\alpha, \beta)_{F, K}^{N}=\Psi_{K}(\alpha)(\widetilde{\beta})-_{F} \widetilde{\beta}
\end{aligned}
$$

где $\widetilde{\beta} \in \widehat{\mathfrak{M}}$ удовлетворяет $\left[\pi^{N}\right]_{F}(\widetilde{\beta})=\beta$.

Символ Гильберта имеет следуюшие свойства.

H.1. $(\cdot, \cdot)_{F, K}^{N}$ аддитивен по первому аргументу и о-линеен по второму.

H.2. $(\alpha, \beta)_{F, K}^{N}=0 \Leftrightarrow \alpha-$ норма в $K_{n}^{\mathrm{top}}(K(\widetilde{\beta}))$. 
Н.3. Если $L / K$ - конечное расширение, то

1) $(\alpha, \beta)_{F, L}^{N}=\left(\alpha, \mathrm{Nm}_{L / K}^{(F)}(\beta)\right)_{F, K}^{N}$ для $\alpha \in K_{n}^{\mathrm{top}}(K), \beta \in F\left(\mathfrak{M}_{L}\right)$, где $\mathrm{Nm}_{L / K}^{(F)}(\beta):=\sum_{\sigma(F)} \sigma(\beta)$

2) $(\alpha, \beta)_{F, L}^{N}=\left(\operatorname{Nm}_{L / K}(\alpha), \beta\right)_{F, K}^{N}$ для $\alpha \in K_{n}^{\operatorname{top}}(L), \beta \in F(\mathfrak{M})$.

H.4. Если $G$ - группа, изоморфная $F$, и $f(x) \in \mathfrak{o}^{\prime}[[\boldsymbol{\Delta}]], f(0)=0,-$ изоморфизм, т.е. $G=f\left(F\left(f^{-1}(x), f^{-1}(y)\right)\right)$, то

$$
(\alpha, \beta)_{G, K}^{N}=f\left(\left(\alpha, f^{-1}(\beta)\right)_{F, K}^{N}\right), \quad \alpha \in K_{n}^{\mathrm{top}}(K), \quad \beta \in G(\mathfrak{M}) .
$$

Доказательства для одномерного случая можно найти в [14], а в многомерном случае они аналогичны.

Нам нужно также обобщение символа Гильберта, которое использует изогению $f^{(N)}($ см. $(4)$ и $[1 ; 1.4])$. А именно рассмотрим спаривание

$$
\begin{aligned}
\{\cdot, \cdot\}_{F}= & \{\cdot, \cdot\}_{F, K}^{N}: K_{n}^{\mathrm{top}}(K) \times F_{N}(\mathfrak{M}) \rightarrow W_{F}^{N} \\
& \{\alpha, \beta\}_{F, K}^{N}=\Psi_{K}(\alpha)(\bar{\beta})-{ }_{F} \bar{\beta}
\end{aligned}
$$

где $f^{(N)}(\bar{\beta})=\beta$.

Нетрудно проверить свойства Н.1-Н.3 для нашего спаривания. Свойство Н.4 будет выплядеть несколько иначе. Пусть $\mathfrak{E}: G \rightarrow F$ - гомоморфизм групп Хонды и $\mathfrak{E}_{N}$ - соответствующий гомоморфизм групп $G_{N}$ и $F_{N}$ из последовательности $(3)$ для $G$ и $F$, т.е. $\mathfrak{E}_{N} \circ g^{(N)}=f^{(N)} \circ \mathfrak{E}_{N}$. Тогда имеем

H. $4^{\prime} .\left\{\alpha, \mathfrak{E}_{N}(\beta)\right\}_{F}=\mathfrak{E}\left(\{\alpha, \beta\}_{G}\right)$.

Кроме того, имеется свойство, которое связьвает два спаривания $(\cdot, \cdot)_{F}$ и $\{\cdot, \cdot\}_{F}:$

H.5. $(\alpha, \beta)_{F}=\left\{\alpha,\left[\pi_{1}^{(N)} / \pi^{N}\right]_{F, F_{N}}(\beta)\right\}_{F}$.

Это свойство вытекает из определений обоих спариваний и равенств

$$
\begin{gathered}
{[a]_{F, F_{N}}=\lambda_{N}^{-1} \circ a \circ \lambda, \quad a \in \mathfrak{o}^{\prime}} \\
f^{(N)}=\lambda_{N}^{-1} \circ \pi_{1}^{(N)} \circ \lambda .
\end{gathered}
$$

\section{§2. Формулировка результатов}

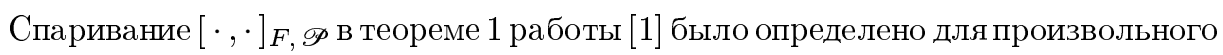
примарного ряда $\mathscr{P}$. Нам нужно выбрать теперь ряд $\mathscr{P}$ так, чтобы получившееся спаривание индуцировало символ Гильберта над $K$. Для этого возьмем в качестве $\mathscr{P}$ ряд $W$ (см. (5)). В п. 3.1.6 ниже мы показываем, что $W$ - примарный ряд в смысле $[1 ; 2.2$, определение 6$]$.

Пусть $z_{1}, \ldots, z_{h}$ - образующие о-модуля $W_{F}^{N}$ и $W_{i}:=\pi_{1}^{(N)} \lambda \circ \underline{z}_{i}$, где $\underline{z}_{i}=\underline{z}_{i}(X)$ - ряд из $\mathfrak{o}_{T}[[X]]_{0}$ такой, что $\underline{z}_{i}\left(t_{1}, \ldots, t_{n-1}, \Pi\right)=z_{i}($ см. (5)). Рассмотрим матрицу

$$
A=\left(\boldsymbol{\Delta}^{i-1} W_{j}\right)_{1 \leqslant i, j \leqslant h}
$$

Положим

$$
\Gamma_{j}=(\operatorname{det} A)^{-1}(-1)^{j+1} A_{j 1},
$$


где $A_{j 1}-$ минор матрицы $A$.

В предложении 3 мы покажем, что $\Gamma_{j}-$ примарный ряд. Определим теперь спаривание

$$
\langle\cdot, \cdot\rangle_{F}: K_{n}\left(\mathscr{H}_{m}\right) \times \mathscr{H}_{F_{N}} \rightarrow W_{F}^{N}
$$

по формуле

$$
\langle\alpha, \beta\rangle_{F}=\sum_{j=1}^{h}(F)\left[\operatorname{Tr}[\alpha, \beta]_{F, \Gamma_{j}}\right]_{F}\left(z_{j}\right),
$$

где $\operatorname{Tr}$ - оператор следа в $T / k$. Пусть $\mathscr{H}_{\text {Eis }}$ - подгруппа в $K_{n}\left(\mathscr{H}_{m}\right)$, порожденная символами $\left\{\alpha_{1}, \ldots, \alpha_{n}\right\}$, для которых хотя бы один из элементов $\alpha_{i}$ лежит в $U_{\mathrm{Eis}}$ (см. $[1 ; 1.6,(16)])$. Пусть, далее, $U_{F_{N}}$ обозначает подгруппу из $\mathscr{H}_{F_{N}}$, определенную в $[1 ; 1.8$, предложение 4$]$.

Теорема 1. Спаривание $\langle\cdot, \cdot\rangle_{F}$ индуцирует спаривание

$\langle\cdot, \cdot\rangle_{F}: K_{n}\left(\mathscr{H}_{m}\right) /\left(\mathscr{H}_{\text {Eis }}+p^{N} K_{n}\left(\mathscr{H}_{m}\right)\right) \times \mathscr{H}_{F_{N}} /\left(U_{F_{N}}+F_{N} f^{(N)}\left(\mathscr{H}_{F_{N}}\right)\right) \rightarrow W_{F}^{N}$, и это спаривание невырождено по второму аргументу.

Используя теорему 1 , мы проверим главный результат работы.

Пусть $\left\{\alpha_{1}, \ldots, \alpha_{n}\right\} \in K_{n}^{\text {top }}(K), \beta \in F(\mathfrak{M})$, и пусть $\underline{\alpha}_{1}, \ldots, \underline{\alpha}_{n}-$ прообразы $\alpha_{1}, \ldots, \alpha_{n}$ в $\mathscr{H}_{m}$, а $\beta$ - прообраз $\beta$ в $\mathscr{H}_{F}($ см. $[1 ; 1.6,(15)]$ и $[1 ; 1.8,(19)])$. Пусть $u=\pi-a_{1} \boldsymbol{\Delta}-\cdots-a_{h} \boldsymbol{\Delta}^{h}-$ канонический тип формальной группы $F$ (см. (2)). Используем функции

$$
\begin{aligned}
& l_{F}(\underline{\beta})=\left(1-\frac{a_{1}}{\pi} \mathbf{\Delta}-\ldots-\frac{a_{h}}{\pi} \mathbf{\Delta}^{h}\right)(\lambda \circ \underline{\beta}), \\
& l_{j}\left(\underline{\alpha}_{i}\right)=\frac{1}{q^{j}} \log \frac{\underline{\alpha}_{i}^{q^{j}}}{\underline{\alpha}_{i}^{\mathbf{\Lambda}^{j}}}
\end{aligned}
$$

(см. $[1 ; 1.9]$ и $[1 ; 2.3,(35)])$. Кроме того, для образующих $z_{1}, \ldots, z_{h}$ o-модуля $W_{F}^{N}$ рассмотрим

$$
S_{j}=\lambda\left(\left[\pi^{N}\right]_{F}\left(\underline{z}_{j}\right)\right) .
$$

ТЕОРема 2. Для обобщенного символа Гильберта имеет место следующая явная формула:

$$
(\alpha, \beta)_{F}=\sum_{k=1}^{h}(F)\left[\operatorname{Tr} \operatorname{res}_{X_{1}, \ldots, X_{n}} \Phi(X) V_{k}(X)\right]_{F}\left(z_{k}\right),
$$

$2 \partial e$

$$
\begin{aligned}
\Phi(X):= & l_{F}(\underline{\beta}) \underline{\alpha}_{1}^{-1} d \underline{\alpha}_{1} \wedge \cdots \wedge \underline{\alpha}_{n}^{-1} d \underline{\alpha}_{n} \\
& -\frac{1}{\pi} \sum_{i=1}^{n} \sum_{j=1}^{h} a_{j} l_{j}\left(\underline{\alpha}_{i}\right) \underline{\alpha}_{1}^{-1} d \underline{\alpha}_{1} \wedge \cdots \wedge \underline{\alpha}_{i-1}^{-1} d \underline{\alpha}_{i-1} \\
& \wedge \frac{1}{q^{j}} d \Lambda^{j} \lambda(\underline{\beta}) \wedge \frac{1}{q^{j}} \underline{\alpha}_{i+1}^{-\mathbf{\Delta}^{j}} d \underline{\alpha}_{i+1}^{\mathbf{\Lambda}^{j}} \wedge \cdots \wedge \frac{1}{q^{j}} \underline{\alpha}_{n}^{-\mathbf{\Delta}^{j}} d \underline{\alpha}_{n}^{\mathbf{\Delta}^{j}}, \\
V_{k}(X)= & (-1)^{k+1} \frac{B_{k 1}}{\operatorname{det} B}, \\
B= & \left(\mathbf{\Delta}^{i-1} S_{j}\right)_{1 \leqslant i, j \leqslant h} .
\end{aligned}
$$




\section{§3. Свойства спаривания $\langle\cdot, \cdot\rangle_{F}$}

\section{1. Корректность определения спаривания $\langle\cdot, \cdot\rangle_{F}$.}

3.1.1. Прежде чем доказывать теорему 1 , мы должны проверить, что спаривание (8) корректно задано. Мы уже убедились, что ряд $\Phi_{N}(\alpha, \beta)$, входяший в определение спаривания $\langle\cdot, \cdot\rangle_{F}$, имеет целые коэффициенты (см. $[1 ; 2.3$, лемма 8]). Следуюшим шагом будет проверка следуюшего предложения.

ПРеДЛОЖЕНИЕ 3. Ряд

$$
\Gamma_{j}=(-1)^{j+1} \frac{A_{j 1}}{\operatorname{det} A}
$$

является примарным в каждом н-иелом модуле

$$
X^{-\bar{e}_{*}} \mathscr{L}_{\mu}, \quad \frac{1}{q^{h}} \leqslant \mu<\frac{q^{h}-1}{q^{2 h-1}} .
$$

(Определения колец $\mathscr{L}$ и $L$ см. в [1;2.1].)

Это предложение не является тривиальньм, и мы должны предварительно доказать несколько вспомогательных результатов.

3.1.2. Главньми объектами нашего исследования являются ряды (и обратные им) вида

$$
D \boldsymbol{\Delta}^{k} W^{(\varkappa)}, \quad D \equiv 1 \quad \bmod \boldsymbol{\Delta} \mathfrak{o}^{\prime}[[\mathbf{\Delta}]],
$$

где $W^{(\varkappa)}=\lambda_{\varkappa}\left(s_{\varkappa}\right)$ (см. (5)). Для $\varkappa=0: \lambda_{0}=\lambda, s_{0}=z$ и $W^{(0)}=\lambda(z)$.

Лемма 1. 1) Ряды $D \boldsymbol{\Delta}^{k} W^{(\varkappa)}$ u $\left(D \boldsymbol{\Delta}^{k} W^{(\varkappa)}\right)^{-1}$ являются главныли единицами в кольце $L_{\mu q^{h(N-\varkappa)-k}}$ степеней $\bar{e}_{*} / q^{h(N-\varkappa)-k} u-\bar{e}_{*} / q^{h(N-\varkappa)-k}$ coomветственно для $\mu \in\left[\frac{1}{q^{h}}, \frac{q^{h}-1}{q^{h}}\right)$. Кроме того, модули

$$
X^{\bar{e}_{*} / q^{h(N-\varkappa)-k}} \mathscr{L}_{\mu q^{h(N-\varkappa)-k}} \quad u \quad X^{-\bar{e}_{*} / q^{h(N-\varkappa)-k}} \mathscr{L}_{\mu q^{h(N-\varkappa)-k}}
$$

являются $\mu$-цельми $\quad \mu$-собственными модулями рядов $\quad D \mathbf{\Delta}^{k} W^{(\varkappa)} \quad u$ $\left(D \boldsymbol{\Delta}^{k} W^{(\varkappa)}\right)^{-1}$ соответственно (cм. определения в $\left.[1 ; 2.1]\right)$.

2) Пусть $W_{i}^{(\varkappa)}=\lambda_{\varkappa}\left(s_{\varkappa}^{(i)}\right)(c \mathcal{M} .(5))$. Тогда рядь

$$
\frac{D \boldsymbol{\Delta}^{k} W_{i}^{(\varkappa)}}{D \mathbf{\Delta}^{k} W_{j}^{(\varkappa)}}, \quad 1 \leqslant i, j \leqslant h,
$$

являются главньми единицами в кольце

$$
\mathscr{L}_{\mu q^{h(N-\varkappa)-k}}, \quad \mu \in\left[\frac{1}{q^{h}}, \frac{q^{h}-1}{q^{h}}\right),
$$

и мы назовем кольцо (10) 
ДокаЗАтельство. Рассмотрим сперва ряд $W:=W^{(N)}=\pi_{1}^{(N)} \lambda(z)($ см. (5)), где $\pi_{1}^{(N)}=\pi^{N} / a_{h}^{1+\Delta^{h}+\cdots+\Delta^{h(N-1)}}$.

Обозначим $1 / a_{h}^{1+\Delta^{h}+\cdots+\Delta^{h(N-1)}}$ через $a \in \mathfrak{o}^{\times}$, тогда согласно [1; 1.4, лемма 2] ряд $W$ можно представить в виде

$$
W=a \pi^{N} \lambda(z)=a\left(\pi^{N} c_{0} z+\pi^{N-1} c_{1} z^{q^{h}}+\pi^{N-2} c_{2} z^{q^{2 h}}+\cdots\right),
$$

где $c_{i} \in \mathfrak{o}^{\prime}[[\mathbf{\Lambda}]]^{\times}$. Отсюда

$$
\begin{aligned}
W= & a z^{q^{N h}}\left(c_{N}+\pi c_{N-1} z^{q^{(N-1) h}-q^{N h}}\right. \\
& \left.+\cdots+\pi c_{0} z^{1-q^{N h}}+\sum_{k \geqslant 1} \frac{c_{N+k}}{\pi^{k}} z^{q^{(N+k) h}-q^{N h}}\right)=a z^{q^{N h}} \cdot \varepsilon(X) .
\end{aligned}
$$

Согласно $[1 ; 1.5$, лемма 3$]$ имеем

$$
a z^{q^{N h}}=b_{0}(X) X^{e_{*}},
$$

где $b_{0} \in \mathfrak{o}_{T}[[X]]^{\times}$и $e_{*}=\frac{q^{h} e}{q^{h}-1}$.

Непосредственно проверяем, что все ряды

$$
\pi^{k} c_{N-k} z^{q^{(N-k) h}-q^{N h}}, \quad 0 \leqslant k \leqslant N,
$$

принадлежат кольцам $\mathscr{L}_{\mu}$ для $\mu \geqslant 1 / q^{h}$, причем нормирование $\nu$ на этих рядах положительно (см. $[1 ; 2.1 .1,(31)])$. А ряды

$$
\frac{c_{N+k}}{\pi^{k}} z^{q^{(N+k) h}-q^{N h}}, \quad k \geqslant 1
$$

лежат в кольцах $\mathscr{L}_{\mu}, \mu \leqslant 1$. Поэтому ряд $\varepsilon(X)$ является главной единицей в кольцах $\mathscr{L}_{\mu}$ для $\mu \in\left[1 / q^{h}, 1\right]$ согласно $[1 ; 2.1 .1$, определение 2]. Поэтому с учетом (11) ряд $W$ будет главной единицей порядка $e_{*}$ в кольцах $\mathscr{L}_{\mu}, \mu \in\left[1 / q^{h}, 1\right]$. Так как главные единицы в кольце $\mathscr{L}_{\mu}$ образуют мультипликативную группу, то ясно, что ряд $W^{-1}$ будет главной единицей порядка $-e_{*}$ в тех же кольцах $\mathscr{L}_{\mu}, \mu \in\left[1 / q^{h}, 1\right]$. Если при этом $\mu<\frac{q^{h}-1}{q^{h}}$, то модуль $X^{-\bar{e}_{*}} \mathscr{L}_{\mu}$ является $\mu$-целым, так как

$$
\mu \bar{e}_{*}<\frac{q^{h}-1}{q^{h}} \bar{e}_{*}=\bar{e}
$$

(см. $[1 ; 2.1 .2$, лемма 6]), и мы проверили второе условие нашей леммы.

Докажем наше утверждение для рядов $D W$ и $(D W)^{-1}, D \equiv 1 \bmod \boldsymbol{\Delta} \mathfrak{o}^{\prime}[[\mathbf{\Lambda}]]$. Записав ряд $c \mathbf{\Delta}^{k} W, c \in \mathfrak{o}^{\prime}$, в виде

$$
c \boldsymbol{\Delta}^{k} W=X^{\bar{e}_{*}} f(X),
$$


нетрудно убедиться, что ряд $f(X) \in \mathscr{L}_{\mu}, \mu \in\left[1 / q^{h}, 1\right]$, и при этом свободный член ряда $f(X)$ делится на $\pi$. Поэтому $\left(1+c \boldsymbol{\Delta}^{k}\right) W$ и, аналогично, $D W$ представим в виде

$$
D W=X^{\bar{e}_{*}} \varepsilon(X),
$$

$\varepsilon(X)$ - главная единица в кольцах $\mathscr{L}_{\mu}, \mu \in\left[1 / q^{h}, 1\right]$, а значит,

$$
(D W)^{-1}=X^{-\bar{e}_{*}} \varepsilon(X)^{-1}
$$

- главная единица порядка $-\bar{e}_{*}$ в кольцах $\mathscr{L}_{\mu}$.

Общий случай леммы проверяется аналогично.

3.1.3. В нашем исследовании ряды

$$
W^{(\varkappa)}=\lambda_{\varkappa}\left(s_{\varkappa}\right), \quad 0 \leqslant \varkappa \leqslant N,
$$

будут играть особую роль.

Используя связь между логарифмами $\lambda_{\varkappa}$ и $\lambda_{\varkappa-1}$ :

$$
\lambda_{\varkappa} \circ f_{\varkappa-1}=\pi_{\varkappa} \circ \lambda_{\varkappa-1}, \quad \pi_{\varkappa}=\frac{\pi}{a_{h}^{\Delta^{h(\varkappa-1)}}}
$$

(см. п. 2) предложения 1 и (4)), мы получим соотношения

$$
W^{(N)}=\pi_{N} W^{(N-1)}=\pi_{N} \pi_{N-1} W^{(N-2)}=\cdots=\pi_{N} \pi_{N-1} \cdots \pi_{1} W^{(0)},
$$

где $W^{(0)}=\lambda(z)$. Пусть

$$
W_{i}:=W_{i}^{(N)}=\lambda_{N}\left(s_{N}^{(i)}\right), \quad 1 \leqslant i \leqslant h,
$$

где $s_{N}^{(i)}=f^{(N)}\left(\underline{z}_{i}\right)$ и $z_{1}, \ldots, z_{h}$ - образуюшие $\mathfrak{o}$-модуля $W_{F}^{N}$. Пусть

$$
\varphi_{i j}=\frac{W_{i}}{W_{j}}, \quad 1 \leqslant i \neq j \leqslant h .
$$

Тогда из леммы 1 получаем

ПРЕДЛОЖЕНИЕ 4. 1) Рядъц $\mathbf{\Delta}^{k} W_{i}, 1 / \mathbf{\Delta}^{k} W_{i}, 0 \leqslant k \leqslant h-1$, являются главными единицами степеней $q^{k} \bar{e}_{*} u-q^{k} \bar{e}_{*}$ соответственно в кольцах $L_{\mu / q^{k}}, \mu \in\left[\frac{1}{q^{h}}, \frac{q^{h}-1}{q^{h}}\right)$. Кроме того, $\mu$-собственнъе модули $X^{q^{k} \bar{e}_{*}} \mathscr{L}_{\mu / q^{k}}$

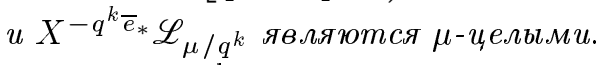

2) Рядьи $\boldsymbol{\Delta}^{k} \varphi_{i j}$ являются главныци единицами в кольцах $\mathscr{L}_{\mu / q^{k}}, 0 \leqslant k \leqslant$ $h-1, \mu \in\left[\frac{1}{q^{h}}, \frac{q^{h}-1}{q^{h}}\right)$.

3) Ряды $\mathbf{\Delta}^{k} W_{i}, 1 / \mathbf{\Delta}^{k} W_{i}, \mathbf{\Delta}^{k} \varphi_{i j}, 0 \leqslant k \leqslant h-1,1 \leqslant i \neq j \leqslant h$, являются главныцми единицами в кольцах $L_{\mu}, \mu \in\left[\frac{1}{q^{h}}, \frac{q^{h}-1}{q^{2 h-1}}\right)$. Кроме того, все эти ряды являются $\mu$-целыми в смысле $[1 ; 2.1 .2$, определение 4]. 
ЗАмечАниЕ 1 . Ряд $W$ может принадлежать различньм типам модулей. Например, согласно (12) имеем

$$
W=\pi_{N} \cdots \pi_{\varkappa+1} W^{(\varkappa)} \in \pi^{N-\varkappa} X^{\bar{e}_{*} / q^{h(N-\varkappa)}} \mathscr{L}_{\mu q^{h(N-\varkappa)}}
$$

где $\mu \in\left[\frac{1}{q^{h}}, \frac{q^{h}-1}{q^{h}}\right)$. Однако при этом он не будет главной единицей в соответствующем кольце $L_{\mu q} q_{(N-\varkappa)}$. Поэтому мы рассматриваем для ряда $W$ только его $\mu$-собственные подмодули.

3.1.4. Пусть $B_{1}, B_{2}, \ldots$ - операторы, соответствующие формальным группам $F, F_{1}, \ldots$ (см. предложение 1 и $\left.(3)\right)$. Пусть $\lambda_{m}$ - логарифм формальной группы $F_{m}$ и $W^{(m)}=\lambda_{m}\left(s_{m}\right)$.

ЛЕмма 2. Имеют место следующие сравнения:

1) $W^{(m)} \equiv B_{m-1} \boldsymbol{\Delta}^{h} W^{(m-1)} \bmod \pi^{m} \mathfrak{o}_{T}[[X]]$,

2) $\left(u_{N} \pi^{-1}\right) W_{i} \equiv 0 \bmod \pi^{N} \mathfrak{o}_{T}[[X]], 1 \leqslant i \leqslant h$, əде $u_{N}$ - канонический тип формальной группь $F_{N}($ см. (3)).

ДоказАтельство. 1) Тип $\widetilde{u}_{m-1}=\pi-\left(\pi / \pi_{m}\right) B_{m-1} \Delta^{h}$ соответствует каноническому типу $u_{m-1}$ формальной группы $F_{m-1}$ и является эквивалентным ему (см. предложение 1). Поэтому

$$
\begin{aligned}
\pi_{1}^{(m)}-B_{m-1} \mathbf{\Delta}^{h} \pi_{1}^{(m-1)} & =\left(\pi_{m}-B_{m-1} \mathbf{\Delta}^{h}\right) \pi_{1}^{(m-1)}=\frac{\pi_{m}}{\pi} \cdot \widetilde{u}_{m-1} \circ \pi_{1}^{(m-1)} \\
& =\frac{\pi_{m}}{\pi} \cdot \pi_{1}^{(m-1)} \widetilde{u}
\end{aligned}
$$

(см. $[1 ; 1.4$, лемма 1, п. (3)]). Остается заметить, что

$$
W^{(m)}-B_{m-1} \mathbf{\Delta}^{h} W^{(m-1)}=\left(\pi_{1}^{(m)}-B_{m-1} \mathbf{\Delta}^{h} \pi_{1}^{(m-1)}\right) \lambda \circ z=\frac{\pi_{m}}{\pi} \cdot \pi_{1}^{(m-1)}(\tilde{u} \circ \lambda) \circ z
$$

И

$$
\widetilde{u} \circ \lambda \equiv 0 \quad \bmod \pi \mathfrak{o}^{\prime}[[X]]
$$

2) Так как $u_{N}$ эквивалентен типу $\widetilde{u}_{N}=\pi-\left(\pi / \pi_{N+1}\right) B_{N} \boldsymbol{\Delta}^{h}$, то достаточно проверить, что

$$
\left(\pi_{N+1}-B_{N} \boldsymbol{\Delta}^{h}\right) W^{(N)} \equiv 0 \quad \bmod \pi^{N+1}
$$

С другой стороны,

$$
\pi_{N+1} W^{(N)}=\pi_{N+1} \pi_{1}^{(N)} \lambda \circ z=\pi_{1}^{(N+1)} \lambda \circ z=W^{(N+1)},
$$

и наше утверждение следует из п. 1).

3.1.5. Пусть $k_{h}$ - неразветвленное расширение степени $h$ поля $k_{c}$, Пусть $\mathfrak{o}_{h}-$ кольцо целых и $\Re_{h}$ - система Тейхмюллера в поле $k_{h}$. Пусть $z_{1}, \ldots, z_{h}$ - образующие о-модуля $W_{F}^{N}$. Аналогично лемме 1.2 из [12] (см. также лемму 4 в [13]) можно проверить следующее утверждение. 
Лемма 3. Существуют әлементы $\theta_{i j} \in \mathfrak{R}_{h}, 1 \leqslant i, j \leqslant h$, такие, что

$$
z_{i} \equiv \theta_{i j} z_{j} \quad \bmod \Pi^{2 e_{n}^{\prime}} \mathfrak{o}_{K} \quad \text { для всех } \quad i, j
$$

где $e_{n}^{\prime}=e_{n} /\left(\left(q^{h}-1\right) q^{h(N-1)}\right)$ (cм. $\left.[1 ; 1.5]\right)$. Кроме того, для кажсого $j$ элементы $\theta_{1 j}, \ldots, \theta_{h j}$ образуют базис о-модуля $\mathfrak{o}_{h}$. Кроме того, $k_{h} \subset T$. Наконеи,

$$
\theta_{i k} \theta_{k j}=\theta_{i j}
$$

ЛЕмма 4. Найдутся әлементы $v_{1}, \ldots, v_{h} \in \mathfrak{o}_{h}$ такие, что для всех $1 \leqslant$ $i, j \leqslant h$ выполнено равенство

$$
\operatorname{Tr}_{h}\left(v_{i} \theta_{j i}\right)=\delta_{i j}
$$

где $\delta_{i j}-$ функиия Мёбиуса, а $\operatorname{Tr}_{h}:=\operatorname{Tr}_{k_{h} / k}$. Кроме того,

1) матрица $\Theta_{k}=\left(\theta_{j k}^{\Delta^{i-1}}\right)_{1 \leqslant i, j \leqslant h}$ обратима и обратной $к$ ней является матрица

$$
\Theta_{k}^{-1}=\left(\left(\theta_{k i} v_{i}\right)^{\Delta^{j-1}}\right)_{1 \leqslant i, j \leqslant h}
$$

2) для любого $b \in \mathfrak{o}_{T}$

$$
\operatorname{Tr}\left(v_{i} \theta_{j i} \widehat{b}\right)=\delta_{i j} \cdot \operatorname{Tr} b
$$

əде $\widehat{b}=b+b^{\Delta}+\cdots+b^{\Delta^{h-1}}$.

ДокАЗАТЕЛЬство. Элемент $v_{i}$ является $i$-м элементом двойственного базиса к $\theta_{1 i}, \theta_{2 i}, \ldots, \theta_{h i}$ относительно спаривания

$$
(x, y) \mapsto \operatorname{Tr}_{h}(x y)
$$

(по предыдушей лемме $\theta_{1 i}, \theta_{2 i}, \ldots, \theta_{h i}$ образуют базис кольца $\mathfrak{o}_{h}$ ).

Далее, по построению элементов $\theta_{j i}$ для всех $1 \leqslant i, j, k \leqslant h$ мы имеем $\theta_{j i} \theta_{i k}=$ $\theta_{j k}$, поэтому

$$
\sum_{m=1}^{h-1}\left(\theta_{i k}^{\Delta^{m}} v_{k}^{\Delta^{m}}\right) \theta_{j i}^{\Delta^{m}}=\sum_{m=0}^{h-1}\left(\theta_{j k} v_{k}\right)^{\Delta^{m}}=\operatorname{Tr}_{h}\left(\theta_{j k} v_{k}\right)=\delta_{k j}
$$

Таким образом, матрица (14) является обратной к $\Theta_{k}$. Кроме того,

$$
\begin{aligned}
\operatorname{Tr}\left(v_{i} \theta_{j i} \widehat{b}\right) & =\operatorname{Tr}_{h} \circ \operatorname{Tr}_{T / k_{h}}\left(v_{i} \theta_{j i} \widehat{b}\right)=\operatorname{Tr}_{h}\left(v_{i} \theta_{j i} \operatorname{Tr}_{T / k_{h}} \widehat{b}\right) \\
& =\operatorname{Tr}_{h}\left(v_{i} \theta_{j i} \operatorname{Tr} b\right)=\operatorname{Tr} b \cdot \operatorname{Tr}_{h}\left(v_{i} \theta_{j i}\right)=\delta_{i j} \operatorname{Tr} b
\end{aligned}
$$

3.1.6. Начиная с этого места, мы фиксируем интервал для $\mu$ :

$$
\mu \in\left[\frac{1}{q^{h}}, \frac{q^{h}-1}{q^{2 h-1}}\right) .
$$

Чтобы проверить корректность задания ряда $\Gamma_{j}($ см. $(9))$, нам нужна следующая 
Лемма 5. Для любого ряда $\varphi_{i j}$ (см. (13)) и любого $k, 0 \leqslant k \leqslant h-1, \boldsymbol{в}$ кольце $\mathscr{L}_{\mu}$ выполнены сравнения:

$$
\Delta^{k} \varphi_{i j} \equiv \theta_{i j}^{\Delta^{k}} \bmod \left(X \mathscr{L}_{\mu}^{+} ; \pi^{N} \mathscr{L}_{\mu}\right)
$$

әде $\theta_{i j} \in \mathfrak{R}^{\times}$, m.е. существуют рядьи $\psi_{k} \in X \mathscr{L}_{\mu}^{+}, \operatorname{deg} \psi_{k}>\overline{0}, u \Lambda_{k} \in \mathscr{L}_{\mu}$, такие, что

$$
\boldsymbol{\Delta}^{k} \varphi_{i j}=\theta_{i j}+\psi_{k}+\pi^{N} \Lambda_{k}
$$

ДоказАтельство. 1) Пусть $D \equiv 1 \bmod \boldsymbol{\Delta} \mathfrak{o}^{\prime}[[\mathbf{\Delta}]]-$ некоторьй оператор в кольце $\mathfrak{o}^{\prime}[[\boldsymbol{\Delta}]]$. Рассмотрим дробь

$$
\frac{D \boldsymbol{\Delta}^{h k} W_{i}}{D \boldsymbol{\Delta}^{h k} W_{j}}, \quad 1 \leqslant k \leqslant N
$$

Вообще говоря, мы можем, используя (12), представить эту дробь различным образом и получать при этом главные единицы в кольцах $\mathscr{L}_{\mu q} q^{h(N-\varkappa-k)}$ (см. лемму 1$)$. Но мы должны использовать только те кольца $L_{\mu}$ и соответствующие им модули, в которых ряды $W_{i}$ и $W_{j}$ являются главньгми единицами. Это возможно тогда и только тогда, когда $\varkappa+k=N$. Поэтому, используя (12), получаем

$$
\begin{aligned}
D \boldsymbol{\Delta}^{h k} W_{i} & =D \boldsymbol{\Delta}^{h k} \pi_{N} \pi_{N-1} \cdots \pi_{N-k+1} W_{i}^{(N-k)} \\
& =\pi^{k} \varepsilon \cdot \lambda_{N-k}\left(D \mathbf{\Delta}^{h k} s_{N-k}^{(i)}\right)=\pi^{k} \varepsilon \cdot D \mathbf{\Delta}^{h k} W_{i}^{(N-k)},
\end{aligned}
$$

где $\pi^{k} \varepsilon=D \boldsymbol{\Delta}^{h k}\left(\pi_{N} \pi_{N-1} \cdots \pi_{N-k+1}\right)$ и $\varepsilon-$ единица в кольце $\mathfrak{o}^{\prime}$ (см. определение $\left.\pi_{N}\right)$.

Применяя лемму 1 , имеем

$$
D \boldsymbol{\Delta}^{h k} W_{i}^{(N-k)}=X^{\bar{e}_{*}} \varepsilon_{i}(X),
$$

где $\varepsilon_{i}(X)$ - главная единица $\mathscr{L}_{\mu}$. Поэтому

$$
\frac{D \mathbf{\Delta}^{h k} W_{i}}{D \mathbf{\Delta}^{h k} W_{j}}=\frac{\pi^{k} \varepsilon \cdot X^{\bar{e}_{*}} \varepsilon_{i}(X)}{\pi^{k} \varepsilon \cdot X^{\bar{e}_{*}} \varepsilon_{j}(X)}=\frac{\varepsilon_{i}(X)}{\varepsilon_{j}(X)} \in \mathscr{L}_{\mu}^{\times} .
$$

Аналогично

$$
\frac{D \boldsymbol{\Delta}^{h} W_{i}}{\pi_{N+1} W_{j}}=\frac{X^{\bar{e}_{*}} \varepsilon_{i}(X)}{X^{\bar{e}_{*}} \eta_{j}(X)}=\frac{\varepsilon_{i}(X)}{\eta_{j}(X)} \in \mathscr{L}_{\mu}^{\times} .
$$

Из (16) и (17) следует, что произведение

$$
\frac{D \boldsymbol{\Delta}^{h} W_{i}}{D \boldsymbol{\Delta}^{h} W_{j}} \cdot \frac{D \boldsymbol{\Delta}^{h} W_{j}}{\pi_{N+1} W_{j}}=\frac{D \boldsymbol{\Delta}^{h} W_{i}}{\pi_{N+1} W_{j}}
$$


корректно определено в кольце $\mathscr{L}_{\mu}$. Полагая $D:=B_{N}($ см. предложение 1 и (3)), получаем, что следующее равенство корректно определено в кольце $\mathscr{L}_{\mu}$ :

$$
\begin{aligned}
\varphi_{i j}= & \frac{W_{i}}{W_{j}}=\frac{B_{N} \boldsymbol{\Delta}^{h} W_{i}}{B_{N} \mathbf{\Delta}^{h} W_{j}} \cdot\left(1+\frac{B_{N} \boldsymbol{\Delta}^{h} W_{j}-\pi_{N+1} W_{j}}{\pi_{N+1} W_{j}}\right) \\
& +\frac{\pi_{N+1} W_{i}-B_{N} \mathbf{\Delta}^{h} W_{i}}{\pi_{N+1} W_{j}} .
\end{aligned}
$$

2) По определению ряда $W_{j}($ см. $(5))$ имеем

$$
\pi_{N+1} W_{j}=\pi_{N+1} \cdot \pi^{(N)} \lambda \circ \underline{z}_{j}=\pi^{(N+1)} \lambda \circ \underline{z}_{j}=W_{j}^{(N+1)} .
$$

Учитывая сравнение 1) леммы 2 и определение $\pi_{N+1}($ см. $(4))$, получаем

$$
\frac{B_{N} \boldsymbol{\Delta}^{h} W_{j}-\pi_{N+1} W_{j}}{\pi_{N+1}}=\frac{B_{N} \boldsymbol{\Delta}^{h} W_{j}-W_{j}^{(N+1)}}{\pi_{N+1}} \in \pi^{N} \mathfrak{o}_{T}[[X]] .
$$

В этом равенстве ряд

$$
\frac{B_{N} \mathbf{\Delta}^{h} W_{j}}{\pi_{N+1}} \in X^{\bar{e}_{*}} \mathscr{L}_{\mu}
$$

так как

$$
B_{N} \boldsymbol{\Delta}^{h} W_{j}=B_{N} \boldsymbol{\Delta}^{h} \pi_{N} W_{j}^{(N-1)}=\pi \varepsilon \cdot B_{N} \boldsymbol{\Delta}^{h} W_{j}^{(N-1)}
$$

и

$$
B_{N} \boldsymbol{\Delta}^{h} W_{j}^{(N-1)} \in X^{\bar{e}_{*}} \mathscr{L}_{\mu}
$$

(см. п. 1) леммы 1). Кроме того, $\frac{\pi_{N+1} \cdot W_{j}}{\pi_{N+1}}=W_{j} \in X^{\bar{e}_{*}} \mathscr{L}_{\mu}$. Поэтому

$$
\frac{B_{N} \boldsymbol{\Delta}^{h} W_{j}-\pi_{N+1} W_{j}}{\pi_{N+1}} \in X^{\bar{e}_{*}} \mathscr{L}_{\mu} \cap \pi^{N} \mathfrak{o}_{T}[[X]]
$$

Отсюда, используя тот факт, что $1 / W_{j} \in X^{-\bar{e}_{*}} \mathscr{L}_{\mu}$ (см. п. 1$)$ леммы 1$)$, получаем

$$
\frac{B_{N} \boldsymbol{\Delta}^{h} W_{j}-\pi_{N+1} W_{j}}{\pi_{N+1} W_{j}} \in \pi^{N} \mathscr{L}_{\mu}
$$

Аналогично

$$
\frac{\pi_{N+1} W_{i}-B_{N} \boldsymbol{\Delta}^{h} W_{i}}{\pi_{N+1} W_{j}} \in \pi^{N} \mathscr{L}_{\mu}
$$

Отсюда и из (18) следует

$$
\varphi_{i j}-\frac{B_{N} \boldsymbol{\Delta}^{h} W_{i}}{B_{N} \boldsymbol{\Delta}^{h} W_{j}} \in \pi^{N} \mathscr{L}_{\mu}
$$

Действуя далее таким же образом, получаем

$$
\frac{B_{N} \boldsymbol{\Delta}^{h} W_{i}}{B_{N} \boldsymbol{\Delta}^{h} W_{j}}-\frac{B_{N+1} \boldsymbol{\Delta}^{h}\left(B_{N} \boldsymbol{\Delta}^{h} W_{i}\right)}{B_{N+1} \boldsymbol{\Delta}^{h}\left(B_{N} \boldsymbol{\Delta}^{h} W_{j}\right)} \in \pi^{N} \mathscr{L}_{\mu}
$$


В результате таких операций имеем

$$
\varphi_{i j}-\frac{B_{2 N} B_{2 N-1}^{\Delta^{h}} \cdots B_{N}^{\Delta_{N}^{h N}} \boldsymbol{\Delta}^{h N} W_{i}}{B_{2 N} B_{2 N-1}^{\Delta^{h}} \cdots B_{N}^{\Delta^{h N}} \boldsymbol{\Delta}^{h N} W_{j}}=\varphi_{i j}-\frac{D \boldsymbol{\Delta}^{h N} W_{i}}{D \boldsymbol{\Delta}^{h N} W_{j}} \in \pi^{N} \mathscr{L}_{\mu}
$$

(напомним, что $\left.\boldsymbol{\Delta} B_{m}=B_{m}^{\Delta} \mathbf{\Delta}\right)$. Кроме того, $D \equiv 1 \bmod \boldsymbol{\Delta} \mathfrak{o}^{\prime}[[\mathbf{\Delta}]]$, так как $B_{m} \equiv 1$ $\bmod \boldsymbol{\Delta}^{\prime}[[\mathbf{\Delta}]]$.

Из равенства (12) получаем

$$
D \mathbf{\Delta}^{h N} W_{i}=\pi^{N} \varepsilon \cdot D \mathbf{\Delta}^{h N} W_{i}^{(0)}=\pi^{N} \varepsilon \cdot D \mathbf{\Delta}^{h N} \lambda\left(z_{i}\right),
$$

где $\pi^{N} \varepsilon=D \boldsymbol{\Delta}^{h N}\left(\pi_{N} \pi_{N-1} \cdots \pi_{1}\right)$ и $\varepsilon$ - единица в $\mathfrak{o}^{\prime}$. По лемме 1

$$
D \boldsymbol{\Delta}^{h N} \lambda\left(z_{i}\right) \in X^{\bar{e}_{*}} \mathscr{L}_{\mu}
$$

Более того, из доказательства этой леммы следует

$$
D \boldsymbol{\Delta}^{h N} \lambda\left(z_{i}\right)=z_{i}^{q^{h N}} \cdot \varepsilon_{i}(X)
$$

где $\varepsilon_{i}(X)$ - главная единица кольца $\mathscr{L}_{\mu}$. Кроме того,

$$
\varepsilon_{i}(X) \in 1+X \mathscr{L}_{\mu}^{+}
$$

Поэтому

$$
\frac{D \mathbf{\Delta}^{h N} W_{i}}{D \mathbf{\Delta}^{h N} W_{j}}=\left(\frac{z_{i}}{z_{j}}\right)^{q^{h N}} \cdot \frac{\varepsilon_{i}(X)}{\varepsilon_{j}(X)} \equiv\left(\frac{z_{i}}{z_{j}}\right)^{q^{h N}} \bmod X \mathscr{L}_{\mu}^{+}
$$

Учитывая лемму 3 , выберем разложение элементов $z_{i}$ и $z_{j}$ в ряды по локальным униформизирующим поля $K$ так, что

$$
\underline{z}_{i} \equiv \theta_{i j} \underline{z}_{j} \quad \bmod X_{n}^{e_{n}^{\prime}} \underline{z}_{j}
$$

Тогда

$$
\frac{D \mathbf{\Delta}^{h N} W_{i}}{D \mathbf{\Delta}^{h N} W_{j}}=\theta_{i j}+\psi, \quad \text { где } \psi \in X \mathscr{L}_{\mu}^{+}, \quad \operatorname{deg} \psi>\overline{0} .
$$

Отсюда и из (19) следует утверждение леммы для ряда $\varphi_{i j}$. Аналогично проверяются соответствующие утверждения для $\boldsymbol{\Delta}^{k} \varphi_{i j}, 0 \leqslant k \leqslant h-1$. 
ДоКАЗАТЕЛЬСТВО ПРЕДЛОЖЕНИЯ 3. 1) Проверим сперва, что модули

$$
X^{-\bar{e}_{*}} \mathscr{L}_{\mu}, \quad \mu \in\left[\frac{1}{q^{h}}, \frac{q^{h}-1}{q^{2 h-1}}\right),
$$

являются $\mu$-собственными модулями для рядов

$$
\Gamma_{j}=(\operatorname{det} A)^{-1}(-1)^{j+1} A_{j 1}
$$

Пусть

$$
\widetilde{A}^{(j)}=\left(\begin{array}{ccc}
\varphi_{1 j} & \ldots & \varphi_{h j} \\
\boldsymbol{\Delta} \varphi_{1 j} & \ldots & \boldsymbol{\Delta} \varphi_{h j} \\
\ldots \ldots \ldots \ldots \ldots & \ldots \ldots \ldots \ldots \\
\boldsymbol{\Delta}^{h-1} \varphi_{1 j} & \ldots & \boldsymbol{\Delta}^{h-1} \varphi_{h j}
\end{array}\right)
$$

где $\varphi_{i j}$ взяты из (13). Тогда $\Gamma_{j}$ можно представить в виде

$$
\Gamma_{j}=\frac{(-1)^{j+1}}{W_{j}} \cdot \frac{\widetilde{A}_{j 1}^{(j)}}{\operatorname{det} \widetilde{A}^{(j)}}
$$

Из леммы 5 следует, что $\operatorname{det} \widetilde{A}^{(j)}$ и $\widetilde{A}_{j 1}^{(j)}$ принадлежат кольцу $\mathscr{L}_{\mu}$. Кроме того,

$$
\operatorname{det} \widetilde{A}^{(j)} \equiv \operatorname{det} \Theta_{j} \bmod \left(X \mathscr{L}_{\mu}^{+} ; \pi^{N} \mathscr{L}_{\mu}\right)
$$

где $\Theta_{j}$ определен в лемме 4 . Согласно лемме $4 \operatorname{det} \Theta_{j}$ обратим в кольце $\mathfrak{o}_{T}$. Поэтому $\operatorname{det} \widetilde{A}^{(j)}$ обратим в кольце $\mathscr{L}_{\mu}$. Отсюда, учитьвая, что $X^{-\bar{e}_{*}} \mathscr{L}_{\mu}$ являются $\mu$-целыми $\mu$-собственными модулями для рядов $1 / W_{j}$ согласно лемме 1 , мы получаем наше утверждение.

2) Проверим теперь, что ряд $\Gamma_{j}$ является примарным в каждом $\mu$-целом $\mu$-собственном модуле $X^{-\bar{e}_{*}} \mathscr{L}_{\mu}$. Для этого докажем прежде всего следующую лемму.

ЛЕмма 6. Ряд $W$ является примарным в каждом $\mu$-иелом $\mu$-собственном модуле $X^{-\bar{e}_{*}} \mathscr{L}_{\mu}$. Кроме того, последовательность

$$
(W)^{(\varkappa)}=D W^{(\varkappa)}, \quad 0 \leqslant \varkappa \leqslant N
$$

где $D=B_{N-1}^{\Delta^{-(N-\varkappa) h}} \cdot B_{N-2}^{\Delta^{-(N-\varkappa-1) h}} \cdots B_{\varkappa}^{\Delta^{-h}}$, примарна для ряда $W$ (операторыл $B_{m}$ определень в предложении 1 и (3)).

ДокАЗАТЕльство. Условие а) из определения для последовательности (23) (см. $[1 ; 2.2$, определение 6]) выполнено согласно лемме 1. Докажем условие b) [1]. Из равенства (12) следует, что

$$
(W)^{(\varkappa)}=D\left(\pi_{\varkappa} \pi_{\varkappa-1} \cdots \pi_{1} \lambda(z)\right)=\pi^{\varkappa} \varepsilon \cdot D \lambda(z),
$$

где $\pi^{\varkappa} \varepsilon=D\left(\pi_{\varkappa} \cdots \pi_{1}\right)$ и $\varepsilon-$ единица в $\mathfrak{o}^{\prime}$. 
По определению операторов $B_{m}$ оператор $D$ можно представить в виде $D=$ $\sum_{m \geqslant 0} d_{m} \boldsymbol{\Delta}^{m}, d_{0}=1, d_{i} \in \mathfrak{o}^{\prime}$. Тогда, используя равенство [1;2.3, $\left.(36)\right]$, получаем

$$
\begin{aligned}
X_{i} \frac{\partial}{\partial X_{i}}\left((W)^{(\varkappa)}\right) & =\frac{\partial}{\partial X_{i}}\left(\pi^{\varkappa} \varepsilon D \lambda(z)\right)=\pi^{\varkappa} \varepsilon \sum_{m \geqslant 0} d_{m} q^{m} \boldsymbol{\Delta}^{m}\left(X_{i} \frac{\partial}{\partial X_{i}}(\lambda(z))\right) \\
& \equiv 0 \bmod \pi^{\varkappa} \mathfrak{o}_{T}[[X]],
\end{aligned}
$$

так как производная логарифма имеет целые коэффициенты. Таким образом, условие b) [1] выполнено.

Проверим, наконец, условие с) [1]. Используя сравнение

$$
W^{(\varkappa)} \equiv B_{\varkappa-1} \boldsymbol{\Delta}^{h} W^{(\varkappa-1)} \quad \bmod \pi^{\varkappa} \mathfrak{o}_{T}[[X]]
$$

(см. п. 1) леммы 2), получаем

$$
\mathbf{\Delta}^{\varkappa}(W)^{(\varkappa)}=B_{N-1}^{\Delta^{-(N-\varkappa) h}} \cdots B_{\varkappa}^{\Delta^{-h}}\left(B_{\varkappa-1} \Delta^{h} W^{(\varkappa-1)}\right) \equiv(W)^{(\varkappa)} \bmod \pi^{\varkappa} \mathscr{L}_{\mu}
$$

(напомним, что $\left.\boldsymbol{\Delta} B_{m}=B_{m}^{\Delta} \mathbf{\Delta}\right)$.

Примарность ряда $\Gamma_{j}$ следует из этой леммы и $[1 ; 2.2$, предложение 8].

ПРЕДЛОЖЕНИЕ 5. Пусть $\Gamma_{j}^{(\varkappa)}$ - примарная последовательность для ряда $\Gamma_{j}($ см. (9)). Тогда

$$
X_{i} \frac{\partial}{\partial X_{i}} \Gamma_{j}^{(\varkappa)}=\sum_{m=0}^{h-1} \pi^{\varkappa+m} X^{-\bar{e}_{*}\left(1+q^{m}\right) / q^{h(N-\varkappa)}} f_{m}(X),
$$

əде $f_{m}(X) \in \mathscr{L}_{\mu q^{h(N-\varkappa)}}, \mu \in\left[\frac{1}{q^{h}}, \frac{q^{h}-1}{q^{2 h-1}}\right)$.

ДокАЗАтЕльство. Мы докажем предложение в случае $\varkappa=N$, т.е. для ряда $\Gamma_{j}$. Остальные случаи рассматриваются аналогично. Ясно, что

$$
X_{i} \partial_{i} \Gamma_{j}=-\Gamma_{j} \frac{1}{\operatorname{det} A} X_{i} \partial_{i}(\operatorname{det} A)+\frac{1}{\operatorname{det} A} \cdot X_{i} \partial_{i} A_{j 1},
$$

где $\partial_{i}:=\partial / \partial X_{i}$

По определению матрицы $A$ имеем

$$
\operatorname{det} A=\sum_{(\alpha) \in S_{n}}(-1)^{J(\alpha)} W_{\alpha_{1}} W_{\alpha_{2}}^{\mathbf{\Delta}} \cdots W_{\alpha_{h}}^{\mathbf{\Lambda}^{h-1}}
$$

Рассмотрим

$$
\psi_{(\alpha)}=\frac{1}{\operatorname{det} A} X_{i} \partial_{i}\left(W_{\alpha_{1}} W_{\alpha_{2}}^{\mathbf{\Delta}} \cdots W_{\alpha_{h}}^{\mathbf{\Delta}^{h-1}}\right)=\frac{1}{\operatorname{det} A} \sum_{j=1}^{h} X_{i} \partial_{i} W_{\alpha_{j}}^{\mathbf{\Delta}^{j-1}} \prod_{\substack{k=1 \\ k \neq j}}^{h} W_{\alpha_{k}}^{\mathbf{\Delta}^{k-1}}
$$


Вьнесем ряд $W_{1}$ из первой строки $\operatorname{det} A$, ряд $W_{1}^{\mathbf{\Delta}}-$ из второй строки и т.д. Тогда получим

$$
\psi_{(\alpha)}=\frac{1}{\operatorname{det} \widetilde{A}} \sum_{j=1}^{h} X_{i} \partial_{i} W_{\alpha_{j}}^{\mathbf{\Delta}^{j-1}} \cdot \frac{1}{W_{1}^{\mathbf{\Delta}^{j-1}}} \prod_{\substack{k=1 \\ k \neq j}}^{h} \varphi_{\alpha_{k}, 1}^{\mathbf{\Lambda}^{k-1}},
$$

где $\varphi_{i j}=W_{i} / W_{j}, \widetilde{A}=\left(\varphi_{i 1}^{\mathbf{A}^{j-1}}\right)_{1 \leqslant i, j \leqslant h}$.

Было доказано, что $(\operatorname{det} \widetilde{A})^{-1} \in \mathscr{L}_{\mu}$ (см. (22)), $\varphi_{i j}^{\mathbf{u}^{m}} \in \mathscr{L}_{\mu}, 0 \leqslant m \leqslant h-1$ (см. предложение 4), $1 / W_{1}^{\mathbf{\Lambda}^{j-1}} \in X^{-q^{j-1} \bar{e}_{*}} \mathscr{L}_{\mu}$ (см. предложение 4).

Кроме того,

$$
X_{i} \partial_{i} W^{\mathbf{\Delta}^{j-1}}=q^{j-1}\left(X_{i} \partial_{i} W\right)^{\mathbf{\Delta}^{j-1}} \equiv 0 \bmod \pi^{N+j-1} \mathfrak{o}_{T}[[X]]
$$

(см. (24)). Поэтому $j$-й член в (26) принадлежит $\pi^{N+j-1} X^{-q^{j-1}} \bar{e}_{*} \mathscr{L}_{\mu}$. Вспоминая, что $\Gamma_{j} \in X^{-\bar{e}_{*}} \mathscr{L}_{\mu}$ (см. предложение 3 ), получаем, наконец,

$$
\Gamma_{j} \frac{1}{\operatorname{det} A} \cdot X_{i} \partial_{i}(\operatorname{det} A)=\sum_{m=0}^{h-1} \pi^{N+m} X^{-\bar{e}_{*}\left(1+q^{m}\right)} g_{m}(X),
$$

где $g_{m}(X) \in \mathscr{L}_{\mu}$.

Действуя аналогично со вторым членом в $(25)$ и применяя при этом определение $A_{j 1}$, получаем

$$
\frac{1}{\operatorname{det} A} X_{i} \partial_{i}\left(A_{j 1}\right)=\sum_{m=0}^{h-1} \pi^{N+m} X^{-\bar{e}_{*}\left(1+q^{m}\right)} h_{m}(X),
$$

где $h_{m}(X) \in \mathscr{L}_{\mu}$. Утверждение предложения следует теперь из (27) и (28).

3.2. Независимость спаривания $\langle\cdot, \cdot\rangle_{F}$ по второму аргументу. В этом пункте мы проверим, что модуль $U_{F_{N}}+F_{N} f^{(N)}\left(\mathscr{H}_{F_{N}}\right)$ содержится в правой части ядра спаривания $\langle\cdot, \cdot\rangle_{F}$ (см. (8)).

ПРЕДЛОЖЕНИЕ 6. Пусть $\beta(X) \in U_{F_{N}}, \alpha(X) \in K_{n}\left(\mathscr{H}_{m}\right)$. Тогда

$$
\langle\alpha(X), \beta(X)\rangle_{F}=0 .
$$

ЗАмечание 2. Мы называем это свойство спаривания $\langle\cdot, \cdot\rangle_{F}$ независимостью. Это название обосновано следующими соображениями. Спаривание (8) естественным образом задает спаривание на множестве $K_{n}^{\text {top }}(K) \times F_{N}(\mathfrak{M})$. А именно пусть $\alpha=\left\{\alpha_{1}, \ldots, \alpha_{n}\right\} \in K_{n}^{\mathrm{top}}(K)$ и $\beta \in F_{N}(\mathfrak{M})$, и пусть $\underline{\alpha}_{1}, \ldots, \underline{\alpha}_{n}$ и $\underline{\beta}-$ прообразы элеметов $\alpha_{1}, \ldots, \alpha_{n}$ и $\beta$ в $\mathscr{H}_{m}$ и $\mathscr{H}_{F_{N}}$ соответственно. Определим тогда

$$
\langle\alpha, \beta\rangle_{F_{N}}:=\langle\underline{\alpha}, \underline{\beta}\rangle_{F} .
$$

Чтобы проверить, что спаривание $\langle\cdot, \cdot\rangle_{F_{N}}$ корректно определено, мы должны показать, что его значение на элементах $\alpha_{1}, \ldots, \alpha_{n}$ и $\beta$ не зависит от выбора прообразов. Поэтому мы называем это свойство независимостью спаривания от способа разложения элементов в степенные ряды по локальным униформизирующим. 
ДОКАЗАТЕЛЬСТво ПРЕДЛОЖЕНИЯ 6. По ПреДПолоЖеНИю $\beta(X) \in U_{F_{N}}$, Поэтомy

$$
\beta(X)=\operatorname{Eis}_{F}(X) \cdot \varphi(X), \quad \varphi \in \mathfrak{o}_{T}[[X]], \quad \varphi(0)=0
$$

(см. $[1 ; 1.8$, предложение 4]). Обозначим временно

$$
\mathscr{E}_{F}:=\operatorname{Eis}_{F}(X) \Longrightarrow \beta=\mathscr{E}_{F} \cdot \varphi
$$

Заметим, что наименшая степень монома ряда $\mathscr{E}_{F}$ и соответственно ряда $\beta$ с обратимьм в кольце $\mathfrak{o}_{T}[[X]]$ коэффициентом не менее $\bar{e}($ см. $[1 ; 1.5$, лемма 3$]$ и $[1$; $1.8,(22)])$.

1) Начнем с проверки следуюшего сравнения.

ПРЕДЛОЖЕНИЕ 7. Пусть

$$
[\alpha, \beta]_{F, \Gamma \varkappa}=\operatorname{res} \Phi_{N}(\alpha, \beta) \cdot \Gamma_{\varkappa}, \quad 1 \leqslant \varkappa \leqslant h
$$

(см. $[1 ; 2.3])$. Тогда

$$
\begin{aligned}
\operatorname{res} & \Phi_{N}(\alpha, \beta) \cdot \Gamma_{\varkappa} \\
& \equiv \operatorname{res} X_{1}^{-1} \cdots X_{n}^{-1}\left(\lambda_{N}(\beta) D \cdot \Gamma_{\varkappa}-\frac{\mathscr{A}(\mathbf{\Lambda})}{\pi}\left(\lambda_{N}(\beta) D \cdot \Gamma_{\varkappa}\right)\right) \bmod \pi^{N},
\end{aligned}
$$

əде

$$
\begin{aligned}
\mathscr{A}(\mathbf{\Lambda}) & =b_{1} \mathbf{\Lambda}+\cdots+b_{h} \mathbf{\Lambda}^{h}, \\
D & =\operatorname{det}\left(X_{j} \alpha_{i}^{-1} \partial_{j} \alpha_{i}\right)_{1 \leqslant i, j \leqslant n}, \\
\Gamma_{\varkappa} & =\frac{(-1)^{\varkappa-1} A_{\varkappa 1}}{\operatorname{det} A} \in X^{-\bar{e}_{*} \mathscr{L}_{\mu},}
\end{aligned}
$$

а ряд $\Phi_{N}(\alpha, \beta)$ определен в $[1 ; 2.3]$.

ЛЕмма 7. Имеет место сравнение

$$
\operatorname{res} \frac{(-1)^{n-i} b_{j}}{\pi q^{j(n-i)}} l_{j}\left(\alpha_{i}\right) D_{i j} \cdot \Gamma_{\varkappa} \equiv-\operatorname{res} \frac{b_{j} \mathbf{\Lambda}^{j}}{\pi} \lambda_{N}(\beta) D_{i j}^{\prime} \cdot \Gamma_{\varkappa} \quad \bmod \pi^{N}
$$

где $D_{i j}$ определены в $[1 ; 2.31]$, a

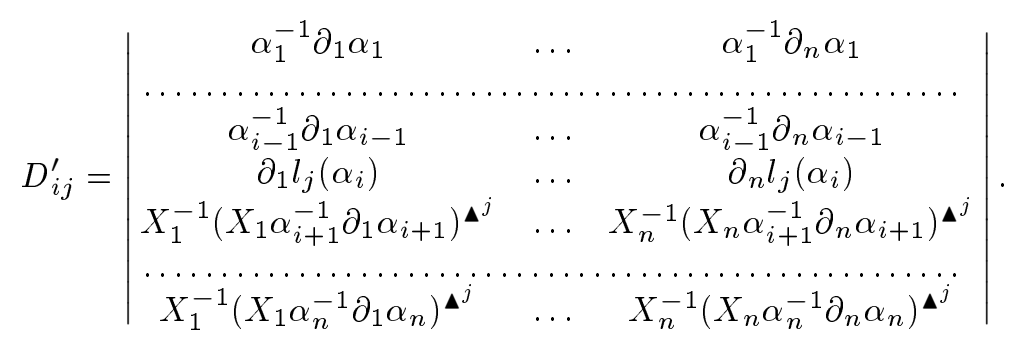


ДокАЗАТЕЛЬСТво. Проверим сравнение

$$
\operatorname{res} l_{j}\left(\alpha_{i}\right) \partial_{k}\left(\frac{b_{j} \mathbf{\Lambda}^{j}}{\pi} \lambda_{N}(\beta)\right) \cdot \Gamma_{\varkappa} \equiv-\operatorname{res} \partial_{k} l_{j}\left(\alpha_{i}\right) \cdot \frac{b_{j} \mathbf{\Lambda}^{j}}{\pi} \lambda_{N}(\beta) \cdot \Gamma_{\varkappa} \quad \bmod \pi^{N}
$$

Заменим сперва ряд $\Gamma_{\varkappa} \bmod \pi^{N}$ на $\left(\Gamma_{\varkappa}^{(N-1)}\right)^{\mathbf{\Lambda}^{h}}$ (см. [1; 2.2, определение 6, п. с)]). Это можно сделать, так как согласно [1;2.3, лемма 8]

$$
l_{j}\left(\alpha_{i}\right) \partial_{k}\left(\frac{b_{j} \mathbf{\Lambda}^{j}}{\pi} \lambda_{N}(\beta)\right) \in \mathfrak{o}_{T}[[X]]
$$

Далее, очевидное равенство res $\partial_{k}(f(X) g(X) h(X))=0$, верное для любых рядов $f, g, h$, показывает, что сравнение (34) равносильно следующему сравнению

$$
\operatorname{res} l_{j}\left(\alpha_{i}\right) \frac{b_{j} \mathbf{\Lambda}^{j}}{\pi} \lambda_{N}(\beta) \cdot \partial_{k}\left(\left(\Gamma_{\varkappa}^{(N-1)}\right)^{\mathbf{\Delta}^{h}}\right) \equiv 0 \bmod \pi^{N}
$$

Из $[1 ; 2.3,(36)]$ получаем тогда

$$
\begin{aligned}
& l_{j}\left(\alpha_{i}\right) \frac{b_{j} \mathbf{\Lambda}^{j}}{\pi} \lambda_{N}(\beta) \cdot \partial_{k}\left(\left(\Gamma_{\varkappa}^{(N-1)}\right)^{\mathbf{\Lambda}^{h}}\right) \\
& \quad=X_{k}^{-1}\left(\frac{q^{h}}{p} l_{j}\left(\alpha_{i}\right)\right) \frac{p}{\pi} \cdot \frac{b_{j} \mathbf{\Lambda}^{j}}{\pi} \lambda_{N}(\beta) \cdot\left(\pi X_{k} \partial_{k}\left(\Gamma_{\varkappa}^{(N-1)}\right)\right) \mathbf{\Delta}^{h} .
\end{aligned}
$$

Имеем два случая: а) $1 \leqslant j \leqslant h-1$; b) $j=h$.

Рассмотрим более сложный случай b) (в случае а) все коэффициенты $b_{j} / \pi$ являются целыми, поэтому он проще). В этом случае $\frac{q^{h}}{p} l_{j}\left(\alpha_{i}\right) \in \mathfrak{o}_{T}[[X]]$ (см. $[1 ; 2.3$, лемма 8]) и $\frac{p}{\pi} b_{j} \in \mathfrak{o}_{T}$. Поэтому достаточно проверить, что ряд

$$
\Psi=\frac{\lambda_{N}(\beta)}{\pi} \cdot \frac{\pi X_{k} \partial_{k}\left(\Gamma_{\varkappa}^{(N-1)}\right)}{\pi^{N}}
$$

является $\mu$-цельм в смысле [1; 2.1.2, определение 4].

По предложению 5 получаем

$$
\Psi=\frac{\lambda_{N}(\beta)}{\pi} \sum_{m=0}^{h-1} \pi^{m} X^{-\bar{e} \frac{q^{m}+1}{q^{h}-1}} f_{m}(X),
$$

где $f_{m} \in \mathscr{L}_{\mu q^{h}}, \mu \in\left[\frac{1}{q^{h}}, \frac{q^{h}-1}{q^{2 h-1}}\right)$.

Осталось доказать, что для каждого $m, 0 \leqslant m \leqslant h-1$, ряд

$$
\pi^{m-1} \lambda_{N}(\beta) \cdot X^{-\bar{e} \frac{q^{m}+1}{q^{h}-1}} f_{m}(X)
$$

является $\mu$-цельм. 
Наименьшая степень монома $\mathscr{E}_{F}$ и, соответственно, ряда $\beta=\mathscr{E}_{F} \cdot \varphi$ с обратимым в кольце $\mathfrak{o}_{T}[[X]]$ коэффициентом не меншше чем $\bar{e}$, поэтому согласно $[1 ; 1.4$, лемма 2$]$ (см. $[1 ; 1.4,(9)])$ ряд $\lambda_{N}(\beta)$ можно представить в виде

$$
\pi^{m-1} \lambda_{N}(\beta)=\pi^{m} a_{0}+\sum_{l \geqslant 1} \pi^{m-l} a_{l} x^{q^{h(l-1)} \bar{e}}
$$

где $a_{i} \in \mathfrak{o}_{T}[[X]]$.

Пусть $f_{m}(X)=\sum d_{\bar{r}} X^{\bar{r}} \in \mathscr{L}_{\mu q^{h}}$. Ряд (37) является $\mu$-цельп, если

a) $\pi^{m} d_{\bar{r}} \in \mathfrak{o}_{T}, \quad \bar{r}<\bar{e} \frac{q^{m}+1}{q^{h}-1}, \quad 0 \leqslant m \leqslant h-1$,

b) $\pi^{m-l} d_{\bar{r}} \in \mathfrak{o}_{T}, \quad \bar{r}<\bar{e}\left(\frac{q^{m}+1}{q^{h}-1}-q^{h(l-1)}\right), \quad 0 \leqslant m \leqslant h-1, l \geqslant 1$.

Заметим, что для $c \in \mathfrak{o}_{T}((\pi))$ выполнено условие

$$
c \in \mathfrak{o}_{T} \Longleftrightarrow v(c) \geqslant 0 .
$$

Пусть $\mu=1 / q^{h}$. Докажем условие (38). По определению кольца $\mathscr{L}_{\mu q^{h}}$ имеем при $q \geqslant 3$

$$
\begin{aligned}
v\left(\pi^{m} d_{\bar{r}}\right) \bar{e} & =m \bar{e}+v\left(d_{\bar{r}}\right) \bar{e} \geqslant m \bar{e}-\mu q^{h} \bar{r} \\
& =m \bar{e}-\bar{r}>\bar{e}\left(m-\frac{q^{m}+1}{q^{h}-1}\right) \geqslant-\frac{2 \bar{e}}{q^{h}-1}>-\bar{e} .
\end{aligned}
$$

Аналогично

$$
\begin{aligned}
v\left(\pi^{m-l} d_{\bar{r}}\right) \bar{e}=( & m-l) \bar{e}+v\left(d_{\bar{r}}\right) \bar{e} \geqslant(m-l) \bar{e}-\bar{r} \\
& >\bar{e}\left(q^{h(l-1)}-l+m-\frac{q^{m}+1}{q^{h}-1}\right) \geqslant-\frac{2 \bar{e}}{q^{h}-1}>-\bar{e} .
\end{aligned}
$$

Таким образом, во всех случаях имеем

$$
v\left(\pi^{m-l} d_{\bar{r}}\right) \bar{e}>-\bar{e}, \quad l, m \geqslant 0 .
$$

Поэтому $v\left(\pi^{m-l} d_{\bar{r}}\right) \geqslant 0$ и, значит, выполнены оба условия (38) и (39), тем самым ряд $\Psi$ (см. (36)) является $\mu$-целым.

Первый случай рассматривается аналогично. Тем самым сравнение (35) и, соответственно, (34) проверены.

Проверим теперь сравнение леммы. Заметим, что

$$
\begin{aligned}
& \frac{(-1)^{n-i} b_{j}}{\pi q^{j(n-i)}} l_{j}\left(\alpha_{i}\right) D_{i j} \cdot \Gamma_{\varkappa} \\
& =(-1)^{n-i} l_{j}\left(\alpha_{i}\right) \cdot \Gamma_{\varkappa}\left|\begin{array}{ccc}
\alpha_{1}^{-1} \partial_{1} \alpha_{1} & \ldots & \alpha_{1}^{-1} \partial_{n} \alpha_{1} \\
\ldots \ldots \ldots \ldots \ldots \ldots \ldots \ldots \ldots \ldots \ldots \ldots \ldots \\
\alpha_{i-1}^{-1} \partial_{1} \alpha_{i-1} & \ldots & \alpha_{i-1}^{-1} \partial_{n} \alpha_{i-1} \\
\frac{1}{q^{j}} \alpha_{i+1}^{-\mathbf{\Delta}^{j}} \partial_{1} \alpha_{i+1}^{\mathbf{\Delta}^{j}} & \ldots & \frac{1}{q^{j}} \alpha_{i+1}^{-\mathbf{\Delta}^{j}} \partial_{n} \alpha_{i+1}^{\mathbf{\Lambda}^{j}} \\
\ldots \ldots \ldots \ldots \ldots & \ldots \ldots & \ldots \ldots \ldots \ldots \\
\frac{1}{q^{j}} \alpha_{n}^{-\mathbf{\Delta}^{j}} \partial_{1} \alpha_{n}^{\mathbf{\Delta}^{j}} & \ldots & \frac{1}{q^{j}} \alpha_{n}^{-\mathbf{\Lambda}^{j}} \partial_{n} \alpha_{n}^{\mathbf{\Delta}^{j}} \\
\partial_{1} \frac{b_{j} \mathbf{\Delta}^{j}}{\pi} \lambda_{N}(\beta) & \ldots & \partial_{n} \frac{b_{j} \mathbf{\Delta}^{j}}{\pi} \lambda_{N}(\beta)
\end{array}\right| .
\end{aligned}
$$


Переставим последнюю строку последовательно на $i$-е место и разложим затем определитель по полученной $i$-й строке. Тогда имеем

$$
\frac{(-1)^{n-i} b_{j}}{\pi q^{j(n-i)}} l_{j}\left(\alpha_{i}\right) D_{i j} \cdot \Gamma_{\varkappa}=(-1)^{n-i} \sum_{k=1}^{n} l_{j}\left(\alpha_{i}\right) \partial_{k}\left(\frac{b_{j} \mathbf{\Delta}^{j}}{\pi} \lambda_{N}(\beta)\right) \cdot \Gamma_{\varkappa} \cdot \Delta_{k},
$$

где $\Delta_{k}$ - минор $k$-го элемента $i$-й строки. Из $[1 ; 2.3,(36)]$ получаем

$$
\frac{1}{q^{j}} \alpha^{-\mathbf{\Delta}^{j}} \partial_{k} \alpha^{\mathbf{\Delta}^{j}}=X_{k}^{-1}\left(X_{k} \alpha^{-1} \partial_{k} \alpha\right)^{\mathbf{\Delta}^{j}} \in \mathfrak{o}_{T}[[X]]
$$

Поэтому $\Delta_{k} \in \mathfrak{o}_{T}[[X]]$. Чтобы закончить доказательство леммы, осталось, используя сравнение (34), разложить определитель в правой части (31) по $i$-й строке.

ДОКАЗАТЕЛЬСТво ПРЕДЛОЖЕНИЯ 7. Рассмотрим первый член ряда $\Phi_{N} \cdot \Gamma_{\varkappa}$ :

$$
l_{F_{N}}(\beta) D_{n+1} \Gamma_{\varkappa}
$$

Используя определение ряда $l_{F_{N}}($ см. $[1 ; 1.9])$, мы можем написать

$$
l_{F_{N}}(\beta) D_{n+1} \Gamma_{\varkappa}=X_{1}^{-1} \cdots X_{n}^{-1} \lambda_{N}(\beta) D \Gamma_{\varkappa}-\frac{\mathscr{A}(\boldsymbol{\Delta})}{\pi} \lambda_{N}(\beta) \cdot \Gamma_{\varkappa} \cdot D_{n+1},
$$

где $D$ определен в (30). Осталось показать, что

$$
\begin{aligned}
& \operatorname{res}\left(\frac{\mathscr{A}(\mathbf{\Delta})}{\pi} \lambda_{N}(\beta) \cdot \Gamma_{\varkappa} \cdot D_{n+1}+\frac{\Gamma_{\varkappa}}{\pi} \sum_{i=1}^{n} \sum_{j=1}^{h} \frac{(-1)^{n-i} b_{j}}{q^{j(n-i)}} l_{j}\left(\alpha_{i}\right) D_{i j}\right) \\
& \quad \equiv \operatorname{res} X_{1}^{-1} \cdots X_{n}^{-1} \frac{\mathscr{A}(\mathbf{\Delta})}{\pi}\left(\lambda_{N}(\beta) D \Gamma_{\varkappa}\right) \bmod \pi^{N} .
\end{aligned}
$$

Отсюда будет немедленно следовать сравнение предложения 7.

Из сравнения леммы 7 вытекает, что второй член в (42) можно переписать в виде $\operatorname{res} \frac{\Gamma_{\varkappa}}{\pi} \sum_{i=1}^{n} \sum_{j=1}^{h} \frac{(-1)^{n-i} b_{j}}{q^{j(n-i)}} l_{j}\left(\alpha_{i}\right) D_{i j} \equiv-\operatorname{res} \sum_{i=1}^{n} \sum_{j=1}^{h} \frac{b_{j} \mathbf{\Delta}^{j}}{\pi} \lambda_{N}(\beta) D_{i j}^{\prime} \cdot \Gamma_{\varkappa} \quad \bmod \pi^{N}$, где $D_{i j}^{\prime}$ задан в $(33)$.

Из $[1 ; 2.3,(36)]$ следует, что

$$
\alpha^{-1} \partial_{k} \alpha-\partial_{k} l_{j}(\alpha)=X_{k}^{-1}\left(X_{k} \alpha^{-1} \partial_{k} \alpha\right)^{\mathbf{\Lambda}^{j}}
$$

Таким образом,

$$
\frac{\mathscr{A}(\mathbf{\Delta})}{\pi} \lambda_{N}(\beta) \cdot \Gamma_{\varkappa} \cdot D_{n+1}-\sum_{j=1}^{h} \frac{b_{j} \mathbf{\Lambda}^{j}}{\pi} \lambda_{N}(\beta) \cdot \Gamma_{\varkappa} \cdot D_{n j}^{\prime}=\sum_{j=1}^{h} \frac{b_{j} \mathbf{\Lambda}^{j}}{\pi} \lambda_{N}(\beta) \cdot \Gamma_{\varkappa} \cdot D_{n}^{(j)}
$$


где

$$
D_{n}^{(j)}=\left|\begin{array}{ccc}
\alpha_{1}^{-1} \partial_{1} \alpha_{1} & \ldots & \alpha_{1}^{-1} \partial_{n} \alpha_{1} \\
\ldots \ldots \ldots \ldots \ldots \ldots \ldots \ldots \ldots \ldots \ldots \ldots \ldots \ldots \ldots \ldots \ldots \ldots \ldots \ldots \ldots \\
\alpha_{n-1}^{-1} \partial_{1} \alpha_{n-1} & \ldots & \alpha_{n-1}^{-1} \partial_{n} \alpha_{n-1} \\
X_{1}^{-1}\left(X_{1} \alpha_{n}^{-1} \partial_{1} \alpha_{n}\right)^{\mathbf{\Delta}^{j}} & \ldots & X_{n}^{-1}\left(X_{n} \alpha_{n}^{-1} \partial_{n} \alpha_{n}\right)^{\mathbf{\Delta}^{j}}
\end{array}\right|
$$

Далее, используя снова $[1 ; 2.3,(36)]$, получаем

$$
\begin{aligned}
& \sum_{j=1}^{h} \frac{b_{j} \mathbf{\Delta}^{j}}{\pi} \lambda_{N}(\beta) \cdot \Gamma_{\varkappa} \cdot D_{n}^{(j)}-\sum_{j=1}^{h} \frac{b_{j} \mathbf{\Delta}^{j}}{\pi} \lambda_{N}(\beta) \cdot \Gamma_{\varkappa} \cdot D_{n-1, j}^{\prime} \\
& \quad=\sum_{j=1}^{h} \frac{b_{j} \mathbf{\Delta}^{j}}{\pi} \lambda_{N}(\beta) \cdot \Gamma_{\varkappa} \cdot D_{n-1}^{(j)},
\end{aligned}
$$

где

$$
D_{n-1}^{(j)}=\left|\begin{array}{ccc}
\alpha_{1}^{-1} \partial_{1} \alpha_{1} & \ldots & \alpha_{1}^{-1} \partial_{n} \alpha_{1} \\
\ldots \ldots \ldots \ldots \ldots \ldots \ldots \ldots \ldots \ldots \ldots \ldots \ldots \ldots \ldots \ldots \ldots \ldots \ldots \ldots \ldots \ldots \ldots \ldots \\
\alpha_{n-2}^{-1} \partial_{1} \alpha_{n-2} & \ldots & \alpha_{n-2}^{-1} \partial_{n} \alpha_{n-2} \\
X_{1}^{-1}\left(X_{1} \alpha_{n-1}^{-1} \partial_{1} \alpha_{n-1}\right)^{\mathbf{\Delta}^{j}} & \ldots & X_{n}^{-1}\left(X_{n} \alpha_{n-1}^{-1} \partial_{n} \alpha_{n-1}\right)^{\mathbf{\Delta}^{j}} \\
X_{1}^{-1}\left(X_{1} \alpha_{n}^{-1} \partial_{1} \alpha_{n}\right)^{\mathbf{\Delta}^{j}} & \ldots & X_{n}^{-1}\left(X_{n} \alpha_{n}^{-1} \partial_{n} \alpha_{n}\right)^{\mathbf{\Delta}^{j}}
\end{array}\right|
$$

Продолжая процесс, получаем

$$
\begin{aligned}
& \operatorname{res}\left(\frac{\mathscr{A}(\mathbf{\Delta})}{\pi} \lambda_{N}(\beta) \cdot \Gamma_{\varkappa} \cdot D_{n+1}-\sum_{i=1}^{n} \sum_{j=1}^{h} \frac{b_{j} \mathbf{\Delta}^{j}}{\pi} \lambda_{N}(\beta) D_{i j}^{\prime} \cdot \Gamma_{\varkappa}\right) \\
& \quad \equiv \operatorname{res} X_{1}^{-1} \cdots X_{n}^{-1} \frac{\mathscr{A}(\mathbf{\Delta})}{\pi}\left(\lambda_{N}(\beta) D \Gamma_{\varkappa}\right) \bmod \pi^{N}
\end{aligned}
$$

что дает нам сравнение предложения 7.

2 ) Очевидно, что ряды $R_{\varkappa}:=\Phi_{N}(\alpha, \beta) \Gamma_{\varkappa}$ являются решениями системы уравнений

$$
A\left(R_{1}, \ldots, R_{h}\right)^{T}=\left(\Phi_{N}(\alpha, \beta), 0, \ldots, 0\right)^{T}
$$

(см. определение $\Gamma_{\varkappa}$ в $\S 2$ ). Мы займемся сейчас преобразованием этой системы.

Рассмотрим следуюшую систему

$$
A\left(\xi_{1}, \ldots, \xi_{h}\right)^{T}=\left(\lambda_{N}(\beta) D,\left(\lambda_{N}(\beta) D\right)^{\mathbf{\Lambda}}, \ldots,\left(\lambda_{N}(\beta) D\right)^{\mathbf{\Lambda}^{h-1}}\right)^{T}
$$

Лемма 8. 1) Решения $\xi_{1}, \ldots, \xi_{h}$ системы (45) являются $\mu$-иельми рядами в кольцах $L_{\mu}, \mu \in\left[\frac{1}{q^{h}}, \frac{q^{h}-1}{q^{2 h-1}}\right)$.

2) Ряды $\xi_{1}^{\mathbf{\Lambda}}, \ldots, \xi_{h}^{\mathbf{\Lambda}}$ являются $\mu$-цельмми рядами в кольцах $L_{\mu / q}\left(\right.$ здесь $L_{\mu}=$ $\bigcup_{\bar{m} \in \mathbb{Z}^{n}} X^{\bar{m}} \mathscr{L}_{\mu}$ ). 
ДокаЗАТЕльство. Наименьшая степень монома ряда $\mathscr{E}_{F}$ с обратимым в кольце $\mathfrak{o}_{T}[[X]]$ коэффициентом равна $\bar{e}($ см. $[1 ; 1.5$, лемма 3$]$ и $[1 ; 1.8,(22)])$. Поэтому точно так же, как в доказательстве леммы 1 , доказываем, что

$$
\lambda_{N}(\beta)^{\mathbf{\Delta}^{m}}=\lambda_{N}\left(\mathscr{E}_{F} \varphi\right)^{\mathbf{\Delta}^{m}} \in X \mathscr{L}_{\mu}^{+}, \quad \mu \geqslant \frac{1}{q^{n}}
$$

Все ряды $\left(X_{k} \alpha^{-1} \partial_{k} \alpha\right)^{\mathbf{\Delta}^{m}}$ имеют целые коэффициенты из о $\mathfrak{o}_{T}[[X]]$ и, значит, все ряды в правой части системы $(45)$ принадлежат кольцу $X \mathscr{L}_{\mu}^{+}$. Тогда, например,

$$
\xi_{1}=\sum_{m=0}^{h-1}(-1)^{m+1}(\lambda(\beta) D)^{\mathbf{\Delta}^{m}} \cdot \frac{A_{1 m}}{\operatorname{det} A}
$$

Действуя точно так же, как при доказательстве предложения 3, мы получаем

$$
\frac{A_{1 m}}{\operatorname{det} A}=\frac{1}{W_{1}^{\mathbf{\Lambda}^{m}}} \cdot \frac{\widetilde{A}_{1 m}}{\operatorname{det} \widetilde{A}}, \quad \widetilde{A}=\left(\varphi_{i 1}^{\mathbf{\Delta}^{k}}\right)_{\substack{1 \leqslant i \leqslant h \\ 0 \leqslant k \leqslant h-1}} .
$$

При этом

$$
\frac{\widetilde{A}_{1 m}}{\operatorname{det} \widetilde{A}} \in \mathscr{L}_{\mu}^{\times}, \quad \mu \in\left[\frac{1}{q^{h}}, \frac{q^{h}-1}{q^{2 h-1}}\right) .
$$

Таким образом,

$$
\frac{A_{1 m}}{\operatorname{det} A} \in X^{-q^{m} \bar{e}_{*}} \mathscr{L}_{\mu}^{\times}
$$

и все модули $X^{-q^{m} \bar{e}_{*}} \mathscr{L}_{\mu}^{\times}, 0 \leqslant m \leqslant h-1$, являются $\mu$-целыми согласно $[1 ; 2.1 .2$, лемма 6] и условию на $\mu$. Поэтому

$$
\xi_{1}=\sum_{m=0}^{h-1} X^{-q^{m} \bar{e}_{*}} f_{m}, \quad f_{m} \in \mathscr{L}_{\mu}
$$

и все ряды $X^{-q^{m} \bar{e}_{*}} f_{m}$ являются $\mu$-цельми, т.е. ряд $\xi_{1}$ является также $\mu$-целым. Аналогично, $\xi_{2}, \ldots, \xi_{h}-\mu$-целые ряды в кольце $L_{\mu}$.

Второе утверждение леммы следует из $[1 ; 2.1 .2$, замечание 11$]$.

Подействуем на первое уравнение системы (45) оператором $b_{1} \boldsymbol{\Delta} / \pi$, на второе уравнение - оператором $b_{2} \boldsymbol{\Delta} / \pi$ и т.д., наконец, на последнее уравнение - оператором $b_{h} \boldsymbol{\Delta} / \pi$ и сложим все уравнения. Тогда получим

$$
\frac{\mathscr{A}(\mathbf{\Delta})}{\pi}\left(W_{1}\right) \xi_{1}^{\mathbf{\Delta}}+\cdots+\frac{\mathscr{A}(\mathbf{\Delta})}{\pi}\left(W_{h}\right) \xi_{1}^{\mathbf{\Delta}}=\frac{\mathscr{A}(\mathbf{\Delta})}{\pi}\left(\lambda_{N}(\beta) D\right)
$$

По определению (см. $[1 ; 1.9])$

$$
l_{F_{N}}(X)=\lambda_{N}(X)-\frac{\mathscr{A}(\mathbf{\Delta})}{\pi}\left(\lambda_{N}(X)\right) .
$$


Поэтому из (47) следует

$$
W_{1} \xi_{1}^{\mathbf{\Delta}}+\cdots+W_{h} \xi_{h}^{\mathbf{\Delta}}=\frac{\mathscr{A}(\mathbf{\Delta})}{\pi}\left(\lambda_{N}(\beta) D\right)+\sum_{i=1}^{h} l_{F_{N}}\left(s_{i}\right) \xi_{i}^{\mathbf{\Lambda}}
$$

Подействуем на первое, второе и т.д., на $(h-1)$-е уравнение системы $(45)$ оператором $\boldsymbol{\Delta}$ и объединим с уравнением (49). Тогда получим систему

$$
A\left(\begin{array}{c}
\xi_{1}^{\mathbf{\Lambda}} \\
\xi_{2}^{\mathbf{\Delta}} \\
\cdots \\
\xi_{h}^{\mathbf{\Delta}}
\end{array}\right)=\left(\begin{array}{c}
\frac{\mathscr{A}(\mathbf{\Delta})}{\pi}\left(\lambda_{N}(\beta) D\right)+\sum_{i=1}^{h} l_{F_{N}}\left(s_{i}\right) \xi_{i}^{\mathbf{\Lambda}} \\
\left(\lambda_{N}(\beta) D\right)^{\mathbf{\Delta}} \\
\cdots \\
\left(\lambda_{N}(\beta) D\right)^{\mathbf{\Delta}^{h-1}}
\end{array}\right)
$$

Вычтем из системы (45) систему (50):

$$
\begin{aligned}
& A\left(\xi_{1}-\xi_{1}^{\mathbf{\Delta}}, \ldots, \xi_{h}-\xi_{h}^{\mathbf{\Delta}}\right)^{T} \\
& \quad=\left(\lambda_{N}(\beta) D-\frac{\mathscr{A}(\mathbf{\Delta})}{\pi}\left(\lambda_{N}(\beta) D\right)-\sum_{i=1}^{h} l_{F_{N}}\left(s_{i}\right) \xi_{i}^{\mathbf{\Lambda}}, 0, \ldots, 0\right)^{T}
\end{aligned}
$$

Из (48) и равенства $\lambda_{N}\left(s_{i}\right)=\pi_{1}^{(N)} \lambda\left(\underline{z}_{i}\right)($ см. (5)) следует, что

$$
\frac{l_{F_{N}}\left(s_{i}\right)}{\pi^{N}} \in \mathfrak{o}_{T}[[X]]
$$

Поэтому решения $r_{1 j}, \ldots, r_{h j}$ системы

$$
A\left(r_{1 j}, \ldots, r_{h j}\right)^{T}=\left(X_{1}^{-1} \ldots X_{n}^{-1} \frac{l_{F_{N}}\left(s_{i}\right)}{\pi^{N}}, 0, \ldots, 0\right)^{T}
$$

принадлежат $\mu$-целому модулю

$$
X^{-\bar{e}_{*}} \mathscr{L}_{\mu}, \quad \mu \in\left[\frac{1}{q^{h}}, \frac{q^{h}-1}{q^{2 h-1}}\right)
$$

(см. предложение 3).

Из системы (53) следует

$$
A\left(\pi^{N} r_{1 j} \xi_{j}^{\mathbf{\Lambda}}, \ldots, \pi^{N} r_{h j} \xi_{j}^{\mathbf{\Lambda}}\right)^{T}=\left(X_{1}^{-1} \cdots X_{n}^{-1} l_{F_{N}}\left(s_{i}\right) \xi_{j}^{\mathbf{\Lambda}}, 0, \ldots, 0\right)^{T}
$$

Поэтому мы имеем систему

$$
A\left(\pi^{N} \sum_{j=1}^{h} r_{1 j} \xi_{j}^{\mathbf{\Lambda}}, \ldots, \pi^{N} \sum_{j=1}^{h} r_{h j} \xi_{j}^{\mathbf{\Lambda}}\right)^{T}=\left(X_{1}^{-1} \cdots X_{n}^{-1} \sum_{j=1}^{h} l_{F_{N}}\left(s_{j}\right) \xi_{j}^{\mathbf{\Lambda}}, 0, \ldots, 0\right)^{T}
$$


Складывая (51) и (54), получаем

$$
\begin{gathered}
A\left(\begin{array}{c}
X_{1}^{-1} \cdots X_{n}^{-1}\left(\xi_{1}-\xi_{1}^{\mathbf{\Lambda}}\right)+\pi^{N} \sum_{j=1}^{h} r_{1 j} \xi_{j}^{\mathbf{\Lambda}} \\
\cdots \\
X_{1}^{-1} \cdots X_{n}^{-1}\left(\xi_{h}-\xi_{h}^{\mathbf{\Lambda}}\right)+\pi^{N} \sum_{j=1}^{h} r_{h j} \xi_{j}^{\mathbf{\Lambda}}
\end{array}\right) \\
=\left(\begin{array}{c}
X_{1}^{-1} \cdots X_{n}^{-1}\left(\lambda_{N}(\beta) D-\frac{\mathcal{A}(\mathbf{\Lambda})}{\pi}\left(\lambda_{N}(\beta) D\right)\right) \\
0 \\
\cdots \\
0
\end{array}\right) .
\end{gathered}
$$

Из полученной системы, определения ряда $\Gamma_{\varkappa}$ и сравнения предложения 7 следует

$$
\operatorname{res} \Phi_{N}(\alpha, \beta) \cdot \Gamma_{\varkappa} \equiv \operatorname{res}\left(X_{1}^{-1} \cdots X_{n}^{-1}\left(\xi_{\varkappa}-\xi_{\varkappa}^{\mathbf{\Delta}}\right)+\pi^{N} \sum_{i=1}^{h} r_{i \varkappa} \xi_{i}^{\mathbf{\Lambda}}\right) \bmod \pi^{N}
$$

При этом все ряды $\xi_{i}, \xi_{i}^{\mathbf{\Delta}}, r_{i \varkappa}$ являются $\mu$-целыми. Таким образом,

$$
\operatorname{Tr} \operatorname{res} \Phi_{N}(\alpha, \beta) \cdot \Gamma_{\varkappa} \equiv \operatorname{Tr} \operatorname{res} X_{1}^{-1} \cdots X_{n}^{-1}\left(\xi_{\varkappa}-\xi_{\varkappa}^{\mathbf{\Delta}}\right)=0 \bmod \pi^{N}
$$

и доказательство предложения 6 закончено.

3.3. Вычисление спаривания $\langle\cdot, \cdot\rangle_{F}$ на базисе Шафаревича. Пусть

$$
\omega(b)(X)=E_{F_{N}}\left(\widehat{b} \lambda_{N}(s)\right)
$$

- ряд, с помошью которого строится примарный элемент $\omega(b)$ в $[1 ; 1.10$, предложение 6], и пусть $\mathscr{E}_{N}^{0}$ и $\mathscr{E}_{N}^{\rho, a}$ - изоморфизмы, определенные в $[1 ; 1.9,(24)]$. Из точной последовательности $[1 ; 1.8$, предложение 4$]$ и из $[1 ; 1.10$, предложение 7$]$ следует, что элементы

$$
\begin{gathered}
\omega_{i}(b)(X), \quad b \in \mathfrak{o}_{T}, \quad 1 \leqslant i \leqslant h, \\
\mathscr{E}_{N}^{0}\left(\theta X_{1}^{i_{1}} \cdots X_{n}^{i_{n}}\right), \quad \theta \in \Re, \quad 0<\bar{\imath} \bar{e}_{*}, \quad p \nmid \bar{\imath}, \quad \text { где } \bar{\imath}=\left(i_{1}, \ldots, i_{n}\right), \\
\mathscr{E}_{N}^{\rho, a}\left(\theta X_{1}^{i_{1}} \cdots X_{n}^{i_{n}}\right), \quad \theta \in \Re, \quad a \in \mathfrak{o}_{T}, \quad 1 \leqslant \rho<f h, \quad 0<\bar{\imath}<\bar{e}_{*}, \quad p \nmid \bar{\imath},
\end{gathered}
$$

порождают систему образующих о-модуля $\mathscr{H}_{F_{N}} \bmod \left(U_{F_{N}}+F_{N} f^{(N)}\left(\mathscr{H}_{F_{N}}\right)\right)$.

ПРЕДЛОЖЕНИЕ 8. 1) Для любъх $\alpha_{i} \in \mathscr{H}_{m}$ имеет место

$$
\left\langle\left(\alpha_{1}, \ldots, \alpha_{n}\right), \omega(b)(X)\right\rangle_{F}=[\delta \operatorname{Tr} b]_{F}(z),
$$

где $\delta$ определен в $[1 ; 1.10$, предложение 6$]$. Кроме того, если один из элементов $\alpha_{1}, \ldots, \alpha_{n}$ является единицей в $\mathscr{H}_{m}$, т.е. он лежит в $\mathfrak{o}_{T}^{\times} U_{m}$, в частности $\left(\alpha_{1}, \ldots, \alpha_{n}\right) \in \mathscr{H}_{\mathrm{Eis}}$, то спаривание тривиально.

2) Имеют место равенства

$$
\begin{gathered}
\left\langle X_{1}, \ldots, X_{n}, \mathscr{E}_{N}^{0}\left(\theta X_{1}^{i_{1}} \cdots X_{n}^{i_{n}}\right)\right\rangle_{F}=0 \\
\left\langle X_{1}, \ldots, X_{n}, \mathscr{E}_{N}^{\rho, a}\left(\theta X_{1}^{i_{1}} \cdots X_{n}^{i_{n}}\right)\right\rangle_{F}=0
\end{gathered}
$$

если $p \nmid \bar{\imath}=\left(i_{1}, \ldots, i_{n}\right)$. 
ДоказАТЕльство. Докажем сперва, что

$$
\left\langle X_{1}, \ldots, X_{n}, \omega(b)(X)\right\rangle_{F}=[\operatorname{Tr} b]_{F}(z)
$$

По определению в $[1 ; 2.3]$ имеем

$$
\Phi_{N}\left(X_{1}, \ldots, X_{n}, \omega(b)(X)\right)=X_{1}^{-1} \cdots X_{n}^{-1} \widehat{b} \lambda_{N}(s)
$$

В системе образующих о-модуля $W_{F}^{N}$ в качестве первой образующей $z_{1}$ возьмем $z$. Тогда получим

$$
\left\langle X_{1}, \ldots, X_{n}, \omega(b)(X)\right\rangle_{F}=\sum_{i=1}^{h} \operatorname{Tr} \operatorname{res}\left(X_{1}^{-1} \cdots X_{n}^{-1} \widehat{b} \lambda_{N}(s) \Gamma_{i}\right)
$$

где

$$
\begin{aligned}
\Gamma_{i} & =\frac{(-1)^{i+1} A_{i 1}}{\operatorname{det} A}=\frac{(-1)^{i+1}}{\lambda_{N}(s)} \cdot \frac{\widetilde{A}_{i 1}}{\operatorname{det} \widetilde{A}} \\
\widetilde{A} & =\left(\begin{array}{ccc}
\varphi_{11} & \ldots & \varphi_{h 1} \\
\varphi_{11}^{\mathbf{A}} & \cdots & \varphi_{h 1}^{\mathbf{\Delta}} \\
\ldots \ldots \ldots & \ldots & \ldots \\
\varphi_{11}^{\mathbf{\Lambda}-1} & \ldots & \varphi_{h 1}^{\mathbf{\Lambda}-1}
\end{array}\right)
\end{aligned}
$$

(см. (21), доказательство предложения 3).

Имеет место следующее сравнение:

$$
\operatorname{det} \widetilde{A} \equiv \operatorname{det} \Theta_{1} \bmod \left(X \mathscr{L}_{\mu}^{+}, \pi^{N} \mathscr{L}_{\mu}\right)
$$

(см. (22)), где $\Theta_{1}$ определен в лемме 4. Обозначив

$$
R_{i}:=\operatorname{res} X_{1}^{-1} \cdots X_{n}^{-1} \widehat{b} \lambda_{N}(s) \Gamma_{i}
$$

мы получим, используя (62),

$$
R_{i} \equiv \widehat{b} \frac{(-1)^{i+1} \Theta_{i 1}}{\operatorname{det} \Theta_{1}} \bmod \pi^{N}
$$

Поэтому $R_{1}, \ldots, R_{h}$ являются решениями системы

$$
\Theta_{1}\left(R_{1}, \ldots, R_{h}\right)^{T} \equiv(\widehat{b}, 0, \ldots, 0)^{T} \bmod \pi^{N}
$$

По лемме 4

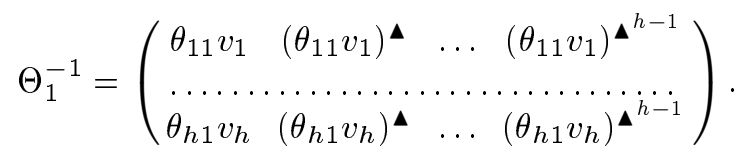

Поэтому

$$
\left(R_{1}, \ldots, R_{h}\right) \equiv\left(\theta_{11} v_{1} \widehat{b}, \ldots, \theta_{h 1} v_{h} \widehat{b}\right) \bmod \pi^{N}
$$


Осталось заметить, что по лемме 4

$$
\operatorname{Tr} \theta_{i k} v_{i} \widehat{b}=\delta_{i k} \operatorname{Tr} b
$$

откуда

$$
R_{1} \equiv \operatorname{Tr} b, \quad R_{2} \equiv \cdots \equiv R_{h} \equiv 0 \quad \bmod \pi^{N},
$$

что завершает доказательство равенства (60).

Пусть теперь один из рядов $\alpha_{1}(X), \ldots, \alpha_{n}(X)$, например $\alpha_{1}(X)=\varepsilon(X)$, является единицей в $\mathscr{H}_{m}$, т.е. $\varepsilon(X) \in U_{m}$ (см. $\left.[1 ; 1.6]\right)$. Проверим, что

$$
\left\langle\varepsilon, \alpha_{2}, \ldots, \alpha_{n}, \omega(b)(X)\right\rangle_{F}=0 .
$$

По формуле из $[1 ; 2.3]$ для спаривания (8) имеем

$$
\Phi_{N} \cdot \Gamma_{k}=s \cdot D_{n+1} \cdot \Gamma_{k}-\left(\frac{1}{\pi} \sum_{i=1}^{n} \sum_{j=1}^{h} \frac{(-1)^{n-i} b_{j}}{q^{j(n-i)}} l_{j}\left(\alpha_{i}\right) D_{i j}\right) \Gamma_{k} .
$$

Для первого слагаемого получаем

$$
s \cdot D_{n+1} \cdot \Gamma_{k}=D_{n+1} \cdot \frac{(-1)^{k+1} \widetilde{A}_{k 1}}{\operatorname{det} \widetilde{A}}
$$

(см. (61)). Как уже говорилось (см. (22)),

$$
\frac{\widetilde{A}_{k 1}}{\operatorname{det} \widetilde{A}} \equiv \frac{\widetilde{A}_{k 1}}{\operatorname{det} \Theta_{1}} \bmod \left(X \mathscr{L}_{\mu}^{+}, \pi^{N} \mathscr{L}_{\mu}\right)
$$

Далее, элементами первой строки определителя $D_{n+1}$ будут ряды $\alpha_{1} \partial_{j} \alpha_{1}=$ $\partial_{j} \log \varepsilon \in \mathfrak{o}_{T}[[X]]$. Поэтому из остальных $n-1$ строк определителя $D_{n+1}$ невозможно получить произведение $X_{1}^{-1} \cdots X_{n}^{-1}$ и, значит,

$$
\operatorname{res} s \cdot D_{n+1} \cdot \Gamma_{k} \equiv 0 \bmod \pi^{N} \text {. }
$$

В остальных определителях $D_{i j}$ все ряды в последней строке делятся на $\pi^{N}$ в кольце $\mathfrak{o}_{T}[[X]]$, так как

$$
\partial_{i}\left(\boldsymbol{\Lambda}^{j} \lambda_{N}(\omega(b)(X))\right) \equiv X_{i}^{-1}\left(q^{i} X_{i} \partial_{i} \lambda_{N}(\omega(b)(X))\right)^{\mathbf{\Lambda}^{i}}
$$

и

$$
\partial_{i} \lambda_{N}(\omega(b)(X)) \equiv 0 \quad \bmod \pi^{N} \mathfrak{o}_{T}[[X]]
$$

(см. $[1 ; 2.3,(36)])$. Отсюда и из $(64)$ следует

$$
\operatorname{res} \Phi_{N} \cdot \Gamma_{k} \equiv 0 \quad \bmod \pi^{N}
$$

что заканчивает доказательство равенства (63).

Равенство (58) следует из (60) и (63) так же, как в доказательстве утверждения $[1 ; 1.10$, предложение 6], с использованием [1; 2.4, предложение 9].

Осталось доказать (59). Пусть $\alpha=\theta X_{1}^{i_{1}} \cdots X_{n}^{i_{n}}, \theta \in \mathfrak{R}, p \nmid \bar{\imath}=\left(i_{1}, \ldots, i_{n}\right)$. Тогда по $[1 ; 2.4$, предложение 9$]$ получаем

$$
\left\langle X_{1}, \ldots, X_{n}, \mathscr{E}_{N}^{0}(\alpha)\right\rangle_{F}=\left\langle X_{1}, \ldots, X_{n-1}, \alpha, \mathscr{E}_{N}^{0}(\alpha)\right\rangle_{F}
$$

(мы предполагаем для простоты, что $p \nmid i_{n}$, в противном случае надо будет поместить элемент $\alpha$ на $k$-е место, для которого $p \nmid i_{k}$ ). Далее мы применяем символьное свойство $[1 ; 2.4$, предложение 9$]$.

Второе равенство проверяется аналогично. 
3.4. Инвариантность спаривания $\langle\cdot, \cdot\rangle$. Инвариантность спаривания означает, что его значение не зависит от выбора локальных униформизирующих. Тем самым мы должны проверить следующее утверждение.

ПрЕДЛОЖЕнИЕ 9. Пусть $Y_{1}, \ldots, Y_{n}$-некоторый набор переменных, и пусть $X_{1}=g_{1}\left(Y_{1}, \ldots, Y_{n}\right), \ldots, X_{n}=g_{n}\left(Y_{1}, \ldots, Y_{n}\right)$. Тогда

$$
\left\langle\alpha_{1}, \ldots, \alpha_{n}, \beta\right\rangle_{F, X}=\left\langle\alpha_{1}, \ldots, \alpha_{n}, \beta\right\rangle_{F, Y}
$$

где $\alpha_{i} \in \mathscr{H}_{m}, \beta \in \mathscr{H}_{F_{N}}$.

ДоказАТЕЛЬСТво. 1) Пусть $\alpha_{1}=X_{1}, \ldots, \alpha_{n}=X_{n}$. Используя независимость спаривания от разложения элементов в ряды, мы можем взять разложение элемента $\beta \in \mathscr{H}_{F_{N}}$ по базису Шафаревича (57) и проверить равенство (65) на образующих базиса Шафаревича.

Пусть сперва $\beta=\omega(b)(X)$ - примарный элемент (56). Тогда

$$
\left\langle X_{1}, \ldots, X_{n}, \omega(b)(X)\right\rangle_{F, X}=[\operatorname{Tr} b]_{F}(z)
$$

(см. (60)). Заметим, что $\omega(b)(X)$ остается примарным элементом в

$$
\mathscr{H}_{F_{N}} \bmod \left(U_{F_{N}+F_{N}} f^{(N)}\left(\mathscr{H}_{F_{N}}\right)\right)
$$

для любого набора переменных (см. $[1 ; 1.10$, предложение 6$])$. Поэтому

$$
\left\langle g_{1}(Y), \ldots, g_{n}(Y), \omega(b)(Y)\right\rangle_{F, Y}=[\operatorname{Tr} b]_{F}(z)
$$

так как $v_{(Y)}\left(g_{1}(Y)\right)=(1,0, \ldots, 0), \ldots, v_{(Y)}\left(g_{n}(Y)\right)=(0, \ldots, 0,1)$. Значит,

$$
\left\langle X_{1}, \ldots, X_{n}, \omega(b)(X)\right\rangle_{F, X}=\left\langle g_{1}(Y), \ldots, g_{n}(Y), \omega(b)(Y)\right\rangle_{F, Y}
$$

Далее,

$$
\left\langle X_{1}, \ldots, X_{n}, \mathscr{E}_{N}^{0}\left(\theta X_{1}^{i_{1}} \cdots X_{n}^{i_{n}}\right)\right\rangle_{F, X}=\left\langle X_{1}, \ldots, X_{n}, \mathscr{E}_{N}^{\rho, a}\left(\theta X_{1}^{i_{1}} \cdots X_{n}^{i_{n}}\right)\right\rangle_{F, X}=0
$$

где $\theta \in \mathfrak{R}, \bar{\imath}=\left(i_{1}, \ldots, i_{n}\right)>\overline{0}, p \nmid \bar{\imath}$ (см. [1; 1.10 , предложение 6$\left.]\right)$. По символьному свойству (см. [1; 2.4, предложение 9, п. 5)]) имеем

$$
\begin{aligned}
& \left\langle g_{1}(Y), \ldots, g_{n}(Y), \mathscr{E}_{N}^{0}\left(\theta g_{1}^{i_{1}} \cdots g_{n}^{i_{n}}\right)\right\rangle_{F, Y} \\
& \quad=\left\langle g_{1}(Y), \ldots, g_{n}(Y), \mathscr{E}_{N}^{\rho, a}\left(\theta g_{1}^{i_{1}} \cdots g_{n}^{i_{n}}\right)\right\rangle_{F, Y}=0,
\end{aligned}
$$

так как $p \nmid \bar{\imath}$. Тем самым (65) проверено для $X_{1}, \ldots, X_{n}$. Заметим, что в доказательстве мы на самом деле использовали независимость спаривания по второму аргументу, так как при переходе к другому набору переменных мы должны были использовать разложение $\beta(Y)$ как формальной суммы образующих $\omega(b)(Y)$, $\mathscr{E}_{N}^{0}\left(\theta g_{1}^{i_{1}} \cdots g_{n}^{i_{n}}\right), \mathscr{E}_{N}^{\rho, a}\left(\theta g_{1}^{i_{1}} \cdots g_{n}^{i_{n}}\right)$. 
2) Обший случай. Символ $\left\{\alpha_{1}, \ldots, \alpha_{n}\right\}$ как элемент $K$-группы можно представить в виде конечной суммы

$$
\left\{\alpha_{1}, \ldots, \alpha_{n}\right\}=\sum_{\bar{Z}} a_{\bar{Z}}\left\{Z_{1}, \ldots, Z_{n}\right\},
$$

где $\bar{Z}=\left(Z_{1}, \ldots, Z_{n}\right)$ - наборы переменных.

Тогда

$$
\begin{aligned}
\left\langle\alpha_{1}, \ldots, \alpha_{n}, \beta\right\rangle_{F, X} & =\sum_{\bar{Z}} a_{\bar{Z}}\left\langle Z_{1}, \ldots, Z_{n}, \beta\right\rangle_{F, X} \\
\left\langle\alpha_{1}, \ldots, \alpha_{n}, \beta\right\rangle_{F, Y} & =\sum_{\bar{Z}} a_{\bar{Z}}\left\langle Z_{1}, \ldots, Z_{n}, \beta\right\rangle_{F, Y}
\end{aligned}
$$

(наборы индексов те же самые). По доказанному вьше

$$
\begin{aligned}
& \left\langle Z_{1}, \ldots, Z_{n}, \beta\right\rangle_{F, X}=\left\langle Z_{1}, \ldots, Z_{n}, \beta\right\rangle_{F, Z}, \\
& \left\langle Z_{1}, \ldots, Z_{n}, \beta\right\rangle_{F, Y}=\left\langle Z_{1}, \ldots, Z_{n}, \beta\right\rangle_{F, Z},
\end{aligned}
$$

откуда немедленно следует равенство (65). Утверждение доказано.

3.5. Независимость спаривания $\langle\cdot, \cdot\rangle_{F}$ по первому аргументу. В этом пункте мы проверим, что группа $\mathscr{H}_{\text {Eis }}+p^{n} K_{n}\left(\mathscr{H}_{m}\right)$ содержится в левой части ядра спаривания $\langle\cdot, \cdot\rangle_{F}($ см. $(8))$.

ПрЕДЛОЖЕНИЕ 10. Пусть $\left(\alpha_{1}, \ldots, \alpha_{n}\right) \in \mathscr{H}_{\text {Eis }}$. Тогда для любого $\beta \in \mathscr{H}_{F_{N}}$ выполнено:

$$
\left\langle\left(\alpha_{1}, \ldots, \alpha_{n}\right), \beta\right\rangle_{F}=0
$$

ДокАЗАТЕльство. Учитывая независимость спаривания $\langle\cdot, \cdot\rangle_{F}$ по второму аргументу (см. предложение 6), достаточно в качестве $\beta$ брать элементы из системы образующих.

Для $\beta=\omega(b)(X)$ равенство (66) следует из предложения 8 , так как по крайней мере один из элементов $\alpha_{1}, \ldots, \alpha_{n}$ лежит в ${ }^{\times} U_{m}$.

Пусть теперь $\beta:=\mathscr{E}_{N}^{0}\left(\theta X^{\bar{\imath}}\right)$, где $\theta \in \Re, X^{\bar{\imath}}=X_{1}^{i_{1}} \cdots X_{n}^{i_{n}}$ и $p \nmid \bar{\imath}$.

Случай 1. $\left(X_{1}, \ldots, \varepsilon(X), \ldots, X_{n}\right)$, где $\varepsilon(X) \in U_{\text {Eis }}$ стоит на $r$-м месте (относительно $U_{\text {Eis }}$ см. $\left.[1 ; 1.6,(16)]\right)$.

Рассмотрим новую систему локальных униформизирующих:

$$
Y=\left(Y_{1}, \ldots, Y_{n}\right), \quad \text { где } Y_{i}=X_{i} \text { при } i \neq r, \quad Y_{r}=X_{r} \varepsilon(X) \text {. }
$$

Пусть $X_{r}=g(Y)=Y \cdot \eta(Y)$, где $\eta(Y) \in U_{m}$. Тогда из инвариантности и из предложения 8 получаем

$$
\begin{aligned}
\left\langle(\ldots, \varepsilon(X), \ldots), \mathscr{E}_{N}^{0}\left(\theta X^{\bar{\imath}}\right)\right\rangle_{F, X} \\
\quad=\left\langle\left(\ldots, X_{r} \varepsilon(X), \ldots\right), \mathscr{E}_{N}^{0}\left(\theta X^{\bar{\imath}}\right)\right\rangle_{F, X}-{ }_{F}\left\langle\left(\ldots, X_{r}, \ldots\right), \mathscr{E}_{N}^{0}\left(\theta X^{\bar{\imath}}\right)\right\rangle_{F, X} \\
\quad=\left\langle\left(Y_{1}, \ldots, Y_{n}\right), \mathscr{E}_{N}^{0}\left(\theta Y_{1}^{i_{1}} \cdots g(Y)^{i_{r}} \ldots Y_{n}^{i_{n}}\right)\right\rangle_{F, Y} .
\end{aligned}
$$


Пусть $X_{i} \rightarrow t_{i}, Y_{i} \rightarrow t_{i}^{\prime}, 1 \leqslant i \leqslant n$, при гомоморфизме [1; 1.6, (14)] (при этом $\left.t_{i}^{\prime}=t_{i}, i \neq r\right)$. Тогда $t_{r}^{\prime}=t_{r} \varepsilon\left(t_{1}, \ldots, t_{n}\right)=t_{r}$, так как $\varepsilon(X) \in U_{\text {Eis }}$. Поэтому

$$
\eta_{m}(g(Y))=t_{r} \quad \text { и } \quad \eta_{m}\left(Y_{r}\right)=t_{r} .
$$

Значит,

$$
\left.\mathscr{E}_{N}^{0}\left(\theta Y^{\bar{\imath}}\right)\right|_{Y=\left(t_{1}^{\prime}, \ldots, t_{n}^{\prime}\right)}=\left.\mathscr{E}_{N}^{0}\left(\theta Y_{1}^{i_{1}} \cdots g(Y)^{i_{r}} \cdots Y_{n}^{i_{n}}\right)\right|_{Y=\left(t_{1}^{\prime}, \ldots, t_{n}^{\prime}\right)}
$$

Тогда из $[1 ; 1.8$, лемма 5$]$ следует

$$
\mathscr{E}_{N}^{0}\left(\theta Y_{1}^{i_{1}} \cdots g(Y)^{i_{r}} \cdots Y_{n}^{i_{n}}\right)=\mathscr{E}_{N}^{0}\left(\theta Y_{1}^{i_{1}} \cdots Y_{r}^{i_{r}} \cdots Y_{n}^{i_{n}}\right)+{ }_{F} \operatorname{Eis}_{F}(Y) \cdot \varphi(Y) .
$$

Используя теперь независимость спаривания $\langle\cdot, \cdot\rangle_{F}$ по второму аргументу (см. предложение 6), мы получаем

$$
\begin{aligned}
& \left\langle Y_{1}, \ldots, Y_{n}, \mathscr{E}_{N}^{0}\left(\theta Y_{1}^{i_{1}} \cdots g(Y)^{i_{r}} \cdots Y_{n}^{i_{n}}\right)\right\rangle_{F, Y} \\
& \quad=\left\langle Y_{1}, \ldots, Y_{n}, \mathscr{E}_{N}^{0}\left(\theta Y_{1}^{i_{1}} \cdots Y_{r}^{i_{r}} \cdots Y_{n}^{i_{n}}\right)\right\rangle_{F, Y}
\end{aligned}
$$

Правая часть последнего равенства равна нулю согласно предложению 8. Поэтому, учитывая (67), имеем

$$
\left\langle\left(X_{1}, \ldots, \varepsilon(X), \ldots, X_{n}\right), \mathscr{E}_{N}^{0}\left(\theta X_{1}^{i_{1}} \cdots X_{n}^{i_{n}}\right)\right\rangle_{F, X}=0
$$

если $p \nmid \bar{\imath}=\left(i_{1}, \ldots, i_{n}\right)$ и $\varepsilon(X) \in U_{\text {Eis }}$.

Значит,

$$
\left\langle\left(X_{1}, \ldots, \varepsilon(X), \ldots, X_{n}\right), \beta(X)\right\rangle_{F, X}=0
$$

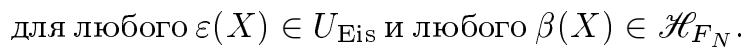

Случай 2. $\left\langle\left(\alpha_{1}, \ldots, \varepsilon(X), \ldots, \alpha_{n}\right), \beta\right\rangle_{F}$.

Представляя символ $\left\{\alpha_{1}, \ldots, \varepsilon(X), \ldots, \alpha_{n}\right\}$ в $K$-группе в виде суммы

$$
\sum_{Z} a_{Z}\left\{Z_{1}, \ldots, \varepsilon(Z), \ldots, Z_{n}\right\}
$$

(перенося $\varepsilon$ в подходящее место, если нужно) и используя инвариантность, мы приходим к случаю 1.

\section{§4. Явные формулы}

4.1. Доказательство теоремы 1. Первая часть теоремы 1 следует из независимости спаривания $\langle\cdot, \cdot\rangle_{F}$ по обоим аргументам, что было проверено в предложениях 6 и 10.

Мы не будем доказьвать невырожденность спаривания по второму аргументу, так как она не используется при доказательстве явной формулы для спаривания Гильберта и доказательство невырожденности аналогично доказательству соответствующего утверждения в мультипликативном случае (см. [15]). 
4.2. Спаривание на формальном модуле $F(\mathfrak{M})$. Из теоремы 1 непосредственно следует, что можно корректно определить следуюшее спаривание:

$$
\langle\cdot, \cdot\rangle_{F}: K_{n}^{\mathrm{top}}(K) \times F(\mathfrak{M}) \rightarrow W_{F}^{N}
$$

А именно пусть $\alpha \in K_{n}^{\mathrm{top}}(K), \beta \in F(\mathfrak{M})$, и пусть $\underline{\alpha} \in K_{n}\left(\mathscr{H}_{m}\right), \underline{\beta} \in \mathscr{H}_{F}-$ их прообразы (см. $[1 ; 1.6,(15)]$ и $[1 ; 1.8,(19)])$. Положим

$$
\langle\alpha, \beta\rangle_{F}:=\langle\underline{\alpha}, \underline{\beta}\rangle_{F}
$$

Из теоремы 1 , а также из независимости и инвариантности спаривания $\langle\cdot, \cdot\rangle_{F}$ (см. предложение 9) следует

ПрЕДЛОЖЕНИЕ 11. Спаривание $\langle\cdot, \cdot\rangle_{F}$ корректно определено, удовлетворяет свойствам 1)-5) [1; 2.4, предложение 9], не зависит от разложения элементов в ряды по локальным параметрам и инвариантно относительно выбора системы локальных параметров.

4.3. Явная формула для символа Гильберта $\{\cdot, \cdot\}_{F}^{N}$.

Теорема 3. Символ Гильберта

$$
\{\cdot, \cdot\}_{F}^{N}: K_{n}^{\mathrm{top}}(K) \times F(\mathfrak{M}) \rightarrow W_{F}^{N}
$$

(см. $[1 ; 1.5])$ совпадает со спариванием $\langle\cdot, \cdot\rangle_{F}$ и тем самым выражсается в явном виде с помощъю формуль (8).

ДокАзАтЕльство. Во-первых, докажем совпадение $\{\cdot, \cdot\}_{F}^{N}$ со спариванием $\langle\cdot, \cdot\rangle_{F}$ на множестве $\left\{t_{1}, \ldots, t_{n}, \beta\right\}$, где $\beta \in F(\mathfrak{M})$, а $t_{1}, \ldots, t_{n}$ - система локальных параметров поля $K$. Ввиду независимости спаривания $\langle\cdot, \cdot\rangle_{F}$ по второму аргументу наше утверждение достаточно проверить на элементах базиса Шафаревича. Последнее следует из [1; 1.10, предложение 7] и предложения 8.

Общий случай. Пусть $\left\{\alpha_{1}, \ldots, \alpha_{n}\right\} \in K_{n}^{\text {top }}(K)$. Тогда

$$
\left\{\alpha_{1}, \ldots, \alpha_{n}\right\}=\sum_{\bar{t}}\left\{t_{1}, t_{2}, \ldots, t_{n}\right\}
$$

- конечная сумма локальных параметров. Далее используем инвариантность спаривания $\langle\cdot, \cdot\rangle_{F}$ и первьй случай.

4.4. Доказательство теоремы 2. Применяя свойство Н.5 и теорему 3 , получаем

$$
\begin{aligned}
(\alpha, \beta)_{F, K}^{N} & =\left\{\alpha,\left[\frac{\pi_{1}^{(N)}}{\pi^{N}}\right]_{F, F_{N}}(\beta)\right\}_{F}=\left\langle\alpha,\left[\frac{\pi_{1}^{(N)}}{\pi^{N}}\right]_{F, F_{N}}(\beta)\right\rangle_{F} \\
& =\sum_{k=1}^{h}(F)\left[\operatorname{Tr} \operatorname{res}_{X_{1}, \ldots, X_{n}} \Phi_{N}\left(\underline{\alpha},\left[\frac{\pi_{1}^{(N)}}{\pi^{N}}\right]_{F, F_{N}}(\underline{\beta)}) \cdot \Gamma_{k}\right]_{F}\left(z_{k}\right),\right.
\end{aligned}
$$

где $\Phi_{N}$ определен в $[1 ; 2.3]$, а $\Gamma_{k}-$ в $(9)$. 
Докажем два равенства

$$
\begin{aligned}
& \Phi_{N}=\frac{\pi_{1}^{(N)}}{\pi^{N}} \cdot \Phi \\
& \Gamma_{k}=\frac{\pi^{N}}{\pi_{1}^{(N)}} \cdot V_{k}
\end{aligned}
$$

Из этих двух равенств и (68) немедленно следует утверждение теоремы.

1) Проверка (69). Из определения $l_{F_{N}}($ см. $[1 ; 1.9])$ и равенств $u_{N} \pi_{1}^{(N)}=\pi_{1}^{(N)} u$ (см. $[1 ; 1.4$, лемма 1, п. $(3)])$ и $[a]_{F, F_{N}}=\lambda_{F_{N}}^{-1}\left(a \lambda_{F}\right)$ следует

$$
\begin{aligned}
(1 & \left.-\frac{b_{1}}{\pi} \mathbf{\Delta}-\ldots-\frac{b_{h}}{\pi} \boldsymbol{\Delta}^{h}\right) \pi_{1}^{(N)}=\left(u_{N} \pi^{-1}\right) \pi_{1}^{(N)} \\
& =\pi_{1}^{(N)}\left(u \pi^{-1}\right)=\pi_{1}^{(N)}\left(1-\frac{a_{1}}{\pi} \boldsymbol{\Delta}-\ldots-\frac{a_{h}}{\pi} \boldsymbol{\Delta}^{h}\right) u
\end{aligned}
$$

Тогда

$$
l_{F_{N}}\left(\left[\frac{\pi_{1}^{(N)}}{\pi^{N}}\right]_{F, F_{N}}(\underline{\beta})\right)=\frac{\pi_{1}^{(N)}}{\pi^{N}} l_{F}(\underline{\beta}) .
$$

Кроме того, $b_{j} \mathbf{\Delta}^{j} \pi_{1}^{(N)}=\pi_{1}^{(N)} a_{j} \mathbf{\Delta}^{j}$ (см. [1; 1.4, лемма 1, п. (3)]), значит,

$$
\begin{gathered}
b_{j} d \mathbf{\Delta}^{j} \lambda_{N}\left(\left[\frac{\pi_{1}^{(N)}}{\pi^{N}}\right]_{F, F_{N}} \underline{(\beta)}\right)=d b_{j} \mathbf{\Delta}^{j}\left(\frac{\pi_{1}^{(N)}}{\pi^{N}}\right) \lambda(\underline{\beta}) \\
=d \frac{\pi_{1}^{(N)}}{\pi^{N}} a_{j} \mathbf{\Delta}^{j} \lambda(\underline{\beta})=\frac{\pi_{1}^{(N)}}{\pi^{N}} a_{j} d \mathbf{\Delta}^{j} \lambda(\underline{\beta}) .
\end{gathered}
$$

Отсюда и из определения $\Phi_{N}$ и $\Phi$ следует (69).

2) Проверка (70). Напомним, во-первых, что

$$
\frac{\pi^{N}}{\pi_{1}^{(N)}} W_{i}=\pi^{N} \lambda \circ \underline{z}_{i}=\lambda\left(\left[\pi^{N}\right]_{F}\left(\underline{z}_{i}\right)\right)=S_{i}
$$

(см. (5)). Значит,

$$
\left(\frac{\pi^{N}}{\pi_{1}^{(N)}}\right)^{\Delta^{m}}\left(\mathbf{\Delta}^{m} W_{i}\right)=\mathbf{\Delta}^{m}\left(\frac{\pi^{N}}{\pi_{1}^{(N)}} W_{i}\right)=\mathbf{\Delta}^{m} S_{i}
$$

Поэтому, умножая числитель ряда $\Gamma_{k}$ на $\left(\pi^{N} / \pi_{1}^{(N)}\right)^{\Delta+\cdots+\Delta^{h-1}}$, а знаменатель на $\left(\pi^{N} / \pi_{1}^{(N)}\right)^{1+\Delta+\cdots+\Delta^{h-1}}$, получаем $(70)$.

Теорема доказана. 


\section{Список литературы}

1. Lorenz F., Vostokov S. V. Honda groups and explicit pairing on the modules of Cartier curves // Algebraic number theory and algebraic geometry / ed. S. Vostokov, Yu. Zarhin. Providence, RI: Amer. Math. Soc., 2002. P. 143-170.

2. Абрашкин A. В. Явная формула для символа Гильберта формальной группы Хонды над векторами Витта // Изв. РАН. Сер. матем. 1997. Т. 61. №3. С. 3-56.

3. Востоков С.В., Демченко О.В. Явная формула для символа Гильберта формальных групп Хонды // Записки научн. сем. ПОМИ. 2000. Т. 272. С. 86-128.

4. Fesenko I., Vostokov S. Local fields and their extensions: a constructive approach. Providence, RI: Amer. Math. Soc., 1993. (Transl. Math. Monogr. V. 121.)

5. Vostokov S. V. Explicit formulas for the Hilbert symbol // Invitation to higher local fields. Geom. Topol. Monogr. 2000. V. 3. P. 81-90.

6. Востоков С. В., Жуков И. Б., Фесенко И.Б. К теории многомерных локальных полей. Методы и конструкции // Алгебра и анализ. 1990. Т. 2. № 4. С. 91-118.

7. Honda T. On the theory of commutative formal groups // J. Math. Soc. Japan. 1970. V. 22. P. 213-243.

8. Демченко О. В. Новое соотношение между формальньми группами Любина-Тейта и формальными группами Хонды // Алгебра и анализ. 1998. Т. 10. № 10. С. 77-84.

9. Париин A. H. Теория полей классов и алгебраическая $K$-теория // УМН. 1975. Т. 30. C. $253-254$.

10. Паршин А.Н. Локальная теория полей классов // Труды МИАН. 1984. Т. 165. C. $143-170$.

11. Kato $K$. A generalisation of local class field theory by using $K$-groups. I // J. Fac. Sci. Univ. Tokyo Sect. IA. 1979. V. 26. P. 303-376; II // J. Fac. Sci. Univ. Tokyo Sect. IA. 1980. V. 27. P. 603-683.

12. Бенуа Д. Г., Востоков С. В. Норменное спаривание в формальных группах и представления Галуа // Алгебра и анализ. 1990. Т. 2. №6. С. 69-97.

13. Демченко О.В. Формальные групшы Хонды: арифметика группы точек // Алгебра и анализ. 2000. Т. 12. №1. С. 132-149.

14. Колывагин В. А. Формалњные групшы и символ норменного выгета // Изв. АН СССР. Сер. матем. 1979. Т. 43. С. 1054-1120.

15. Востоков С. В. Явная конструкция теории полей классов для многомерных локалњных полей // Изв. АН СССР. Сер. матем. 1985. Т. 49. С. 283-308.

Санкт-Петербургский государственный университет; Поступила в редакцию University of Münster, Germany 04.06 .2002

E-mail: sergei@vostokov.usr.pu.ru; lorenz@math.uni-muenster.de 